\title{
Die Rückkehr des Bilderstreites ins Recht
}

\author{
Quid tum? \\ (Leon Battista Alberti)
}

Kehren die Bilder ins Recht zurück?' ${ }^{1}$ Der moderne Rechtsstaat war ikonophob. Die Sprachen des Rechts und die Welt der Bilder scheinen im Hinblick auf die Virtualisierung des Rechts im Internet, auf die Symbolisierungstendenzen postnationaler Rechtsregimes und auf den Skandalisierungsdruck der Völkerrechtspolitik vermehrt Allianzen einzugehen. $^{2}$ Es wird gesagt, "die auf der Basis von gedruckter Schrift entwickelten Konzeptionen der Kommunikationsfreiheit" erschienen "angesichts der Medienentwicklung im post-gutenbergschen Zeitalter überholt". ${ }^{3}$ Waren die Bilder jemals verschwunden? Wenn von einer Rückkehr des Bilderstreites ins Recht gesprochen werden kann, dann, weil das Recht sich in neue Ambivalenzen zwischen Regulierung und Darstellung begibt und damit neue Zweifel an seiner Eigenrationalität

\footnotetext{
${ }^{1} \mathrm{Vgl}$. aus den zahlreichen Publikationen zu Bild und Recht unter anderem COSTAS DOUZINAS/LYNDA NEAD (Hrsg.), Law and the Image. The authority of Art and the Aesthetics of Law. Chicago 1999; PIERRE LEGENDRE, Dieu au mirroir: Etude sur l'institution des images. Paris 1994; PETER GOODRICH, Oedipus Lex. Psychoanalysis, History, Law. Berkeley 1995; Michael Stolleis, Das Auge des Gesetzes. Die Geschichte einer Metapher. München 2004 GERTRUD KOCH/SYLVIA SASSE/LUDGER SCHWARTE (Hrsg.), Kunst als Strafe. Zur Ästhetik der Disziplinierung. München 2003; LUDGER SCHWARTE/ CHRISTOPH WULF (Hrsg.), Körper und Recht. Anthropologische Dimensionen der Rechtsphilosophie. München 2003; MARIETHERES FOGEN, Römische Rechtsgeschichten. Über Ursprung und Evolution eines sozialen Systems. Göttingen 2003; GÜNTHER FRANKENBERG/PETER NIESEN (Hrsg.), Bilderverbot. Recht/Ethik und Ästhetik der öffentlichen Darstellung. Münster 2004; KLAUS RÖHL, Das Recht nach der visuellen Zeitenwende, in: JZ 2003, 339; FABIAN STEINHAUER, Who's afraid of black, red and gold? Zur Geburt der Ikonophobie aus dem Geist des Kriegsrechts, in: WERNER ERNST (Hrsg.), Aufspaltung und Zerstörung durch disziplinäre Wissenschaften. Innsbruck 2003, 79; RONNIE LIPPENS/WOUTER WERNER, Images and Narratives of International Law And Regulation, in: International Journal for the Semiotics of Law 17 $(2004), 123$.

2 Zur Virtualisierung des Rechts siehe RÖHL, Das Recht nach der visuellen Zeitenwende (Fn. 1); zu Symbolisierungstendenzen siehe ULRICH HALTERN, Gestalt und Finalität, in: ARMIN V. BOGDANDY (Hrsg.), Europäisches Verfassungsrecht. Theoretische und dogmatische Grundzïge. Berlin 2002, 803; zum Skandalisierungsdruck siehe ANDREAS FISCHERLESCANO, Die Emergenz der Globalverfassung, in: ZaöRV 63 (2003), 717.

${ }^{3}$ So FRANKENBERG/NIESEN, Bilderverbot (Fn, 1) in ihrer Einleitung.
} 
entstehen. Der vorliegende Beitrag erzählt weder eine Rechtsgeschichte noch eine Bildgeschichte, vor allem aber keine Geschichte abnehmender oder zunehmender Bilddichte im Recht. Bilder sind - wie der Mond - auch dann vorhanden, wenn sie nicht beleuchtet werden. Hier wird eine Geschichte des Streites um Bilder erzählt, dessen Bedingungen sich für Bilder und Recht in der Moderne gleichermaßen geändert haben und weiter ändern. Dies erfordert zunächst einige allgemeine Anmerkungen zu Kommunikation und Medien im Recht.

\section{Vom Stufenbau zum Stereoeffekt - Kommunikation und Medien im Recht}

Kommunikation und Medium haben sich im 20. Jahrhundert zu wissenschaftsführenden Orientierungshilfen entwickelt. Kommunikation wird als Emergenz des Sozialen schlechthin gedacht. ${ }^{4}$ Medien werden als der Speicher eines globalen Gedächtnisses oder als Kristallisationsform des Sozialen vorgestellt. ${ }^{5}$ Das $u b i$ societas, ibi ius steht damit in Konkurrenz zum ubi societas, ibi communicatio - als wären beide Sozialisationsformen bis zur Verdrängung hin aufeinander angewiesen. Sowohl der Begriff der Kommunikation als auch jener des Mediums haben eine ähnlich weit reichende Bedeutung in der wissenschaftlichen Selbstverständigung, wie der Begriff der Kausalität im 19. Jahrhundert. Sie prägen heute vermutlich in ähnlicher Weise das Theoriedesign der Rechtswissenschaft, wie es einst der aus der Naturwissenschaft entlehnte Begriff

\footnotetext{
${ }^{4}$ Dazu u. a. GEORGE HERBERT MEAD, Mind, Self and Society from the Standpoint of a Social Behaviorist (1934). Deutsch Frankfurt am Main 1968; CLAUDE E. SHANNON/WARREN WEAVER, The Mathematical Theory of Communication (1949). Champaign (Il.) 1999; JÜRGEN RUESCH/GREGORY BATESON, Communication. The Social Matrix of Psychiatry. Toronto 1951; MICHEL SERRES, Hermès I. La Communication. Paris 1968; JÜRGEN HABERMAS, Theorie des kommunikativen Handelns. Frankfurt am Main 1981; NIKLAS LUHMANN, Was ist Kommunikation? in: FRITZ B. SIMON (Hrsg.), Lebende Systeme. Wirklichkeitskonstruktion in der systemischen Therapie. Berlin 1988, DERS., Die Gesellschaft der Gesellschaft. Frankfurt am Main 1997; DIRK BAECKER, Kommunikation im Medium der Information, in: Wozu Systeme? Berlin 2002, 111; DERS. Kommunikation, in: KarLHEINZ BARCK/MARTIN FONTIUS/DIETER SCHLENSTEDT/BURKHART STEINWACHS/FRIEDRICH WOLFZETTEL (Hrsg.), Ästhetische Grundbegriffe. Bd. 3. Stuttgart 2001, 384.

5 Vgl. MARSHALl MCLUHAN, Understanding Media. The Extensions of Man. New York 1965, 57; TALCOTT PARSONS, Social Structure and the Symbolic Media of Interchange, in: P.M. BLAU (Hrsg.), Approaches to the Study of Social Structure. London 1976, 94; FRIEDRICH KITTLER, Grammophon, Film, Typewriter. Berlin 1986; HAROLD INNIS, Empire and Communication. Victoria 1986; JOCHEN SCHULTE-SASSE, Medien/medial, in: KARLHEINZ BARCK/MARTIN FONTIUS/DIETER SCHLENSTEDT/BURKHART STEINWACHS/FRIEDRICH WOLFZETTEL (Hrsg.), Ästhetische Grundbegriffe. Bd. 4. Stuttgart 2001, 1; JAN ASSMANN Das kulturelle Gedächtnis. München 1999.
} 
tat. Die Front zwischen Materialismus und Idealismus im 19. Jahrhundert, anhand derer auch die Fronten von Positivismus und Naturrecht oder Begriffs- und Interessensjurisprudenz weiter entfaltet werden konnten, ist noch weit gehend von einem Begriff der Kausalität bestimmt, von dem aus "letzte Instanzen", Einflussgeber und Hintermänner bzw. Hintersubjekte z. B. des Rechts benannt wurden. ${ }^{6}$ Es waren schließlich seine stabilisierenden Möglichkeiten, die den Begriff so attraktiv machten. Egal wohin das Recht auch floss, die Quelle war immer am gleichen Ort, und Kausalität war die Vorstellung, dorthin zurückkehren zu können. Noch der Begriff der Zurechnung in der Reinen Rechtslehre von Hans Kelsen war eben ein echter Ersatzbegriff zum Begriff der Kausalität, indem mit ihm das Wirkungsverständnis von Anstoß und Reaktion in Form von Anlass und Interpretation übernommen und auf die Grundnorm zurückgeführt wurde. ${ }^{7}$

Es ist zweifelhaft, ob in dieser Form die Naturwissenschaft dem Recht noch Ver(antw)ortung zur Verfügung stellen kann. Mit Kommunikation/Medien gerät man heute zwischen die Fronten des 19. Jahrhunderts. Eine Analyse des Rechts unter dem Aspekt der Kommunikation beobachtet nicht die Beziehung von Ursache und Wirkung, sondern kontingente und wechselwirkende Abhängigkeiten zwischen einer Vielzahl von Kommunizierenden und ihren medientechnisch archivierten Beständen. ${ }^{8}$ Kommunikation, so der minimale gemeinsame Nenner der Kommunikationstheorien nach Shannon und Weaver, ist wechselseitige Selektion in einem sozial bestimmbaren Möglichkeitsraum. Schon mit dieser fast bescheiden klingenden Vorgabe ist es nur schwer möglich, die Dynamik des Rechts in Analogie zu Quelle und Einflüssen zu beschreiben und auf ein Letztes zurückzuführen. ${ }^{9}$ Die Metaphorik

${ }^{6}$ Dazu: RUDOLF WIETHÖLTER; Begriffs- und Interessenjurisprudenz - falsche Fronten im IPR, in: ALEXANDER LÜDERITZ (Hrsg.), Internationales Privatrecht und Rechtsvergleichung im Ausgang des 20. Jahrhunderts: Bewahrung oder Wende? Frankfurt am Main 1977, 213 GUNTHER TEUBNER, Der Umgang mit Rechtsparadoxien: Derrida, Luhmann, Wiethölter, in: CHRISTIAN JOERGES/GUNTHER TEUBNER (Hrsg.) Rechtsverfassungsrecht: Rechtfertigung zwischen Prioatrechtsdogmatik und Gesellschaftstheorie. Baden-Baden 2003, 25.

7 HANS KELSEN, Reine Rechtslehre (1934). Aalen 1994, 25 ff., 90; dazu auch das Vorwort zum Neudruck von STANLEY L. PAULSON, VI $\mathrm{f}$.

${ }^{8}$ Zum Begriff des Archivs siehe WOLFGANG ERNST, Das Rumoren der Archive. Ordnung aus Unordnung. Berlin 2002, 10 f., 15-21 mit Verweisen auf Foucaults Vorstellung, dass „weder die Summe aller überlieferten Dokumente noch die Institution“ das Archiv sei, sondern das System, das das "Auftauchen sowie das weitere Funktionieren der Aussagen regiert." Ernst spricht von einem kybernetischen Archivbegriff, nach dem das Archiv ein Dispositiv bzw. einen submedialen Trägerraum darstelle; dazu auch: BORIS GROYS, Unter Verdacht. Eine Phänomenologie der Medien. München 2000.

9 Zu Kritik und Verteidigung des Begriffs Rechtsquelle siehe zuletzt MARKUS KALTENBORN, Gibt es einen Numerus Clausus der Rechtsquellen?, in: Rechtstheorie 34 (2003), 459; zur weiteren Unterscheidung zwischen dem Begriff der Rechtsquelle und anderen 
wandelt sich vom Stufenbau zum Stereoeffekt. Die Orientierung der Wissenschaften an Kategorien der Kommunikations- und Medientheorie hat erhebliche Auswirkungen, vor allem auf die Modelle der Rechtswissenschaft, in denen ein unbewegter Beweger wie Volk, Gesetzgeber, Grundnorm oder der Richter Rechtsentwicklungen anstößt. Vor allem also sind es die Modelle wie das (zivilrechtliche) Regel-Anwendungsmodell oder das aus öffentlich-rechtlicher Tradition stammende Modell parlamentarischer Legitimation, ${ }^{10}$ die vor diesem Hintergrund und einhergehend mit der modernen Schwäche der Subjektphilosophie umgeschrieben werden oder zumindest unter erheblichem Übersetzungsdruck stehen. ${ }^{11}$

Diese Entwicklung betrifft reflexiv auch die Theorien juristischer Kommunikation selbst. Rechtskommunikation, ob schriftlich, mündlich oder bildlich und wie rhetorisch und theatralisch auch immer, ist nur schwer als Einfluss auf das Recht zu beschreiben, das sich abseits der Kommunikationsperspektive anders entwickelt oder entwickeln müsste. Der Begriff des Einflusses ist schließlich von der Vorstellung abhängig, Kommunikation sei entweder ein trojanisches Pferd oder eine Transversale, die dem „Außen“ (z. B. Gesellschaft oder Politik) erlaubt, in das "Innen" (Rechtsgemeinschaft) zu gelangen. Der Umstand, dass Recht kommuniziert wird, kann selbst aber weder die autonome Geschlossenheit des Rechts noch die gesellschaftskritische Offenheit seiner Interpreten garantieren. Kommunikation erlaubt weder einen kontrollierten Ausstieg noch einen kontrollierten Einstieg ins Recht. Kommunikation ist kein Sonderfall des Rechts. Und - das folgt eben aus den Bedingungen wechselseitiger Selektionen - Recht ist kein Sonderfall der Kommunikation. Wie ist dann damit umzugehen, dass sich mit den Kommunikationsbedingungen auch die Bedingungen des Rechtes ändern? Dafür gibt es verschiedene Antworten und das heißt verschiedene Möglichkeiten, mit Mitteln der Kommunikation Grenzen des Rechts zu ziehen. Im vorliegenden Beitrag sollen dabei nur be-

Einheitssymbolen: NiKLAS LuHMANN, Das Recht der Gesellschaft. Frankfurt am Main 1995, 98; DERS., Die juristische Rechtsquellenlehre aus soziologischer Sicht, in: Ausdifferenzierung des Rechts. Frankfurt am Main 1999, 308.

${ }^{10}$ So exemplarisch unter Rückgriff auf die Vorstellung der Kausalität noch in der Formel des Bundesverfassungsgerichtes, in einer Demokratie solle sich die Willensbildung vom Volk zu den Staatsorganen, nicht umgekehrt von den Staatsorganen zum Volk vollziehen, BVerfGE 20,56 (98 f.).

11 Siehe u. a. KARL-HEINZ LADEUR, Postmoderne Rechtstheorie, Berlin 1992, 33-50; GUNTHER TEUBNER, Des Königs viele Leiber. Die Selbstdekonstruktion der Hierarchie des Rechts, in: Soziale Systeme 2, 1996, 229-255; DERS., Globale Bukowina. Zur Emergenz eines transnationalen Rechtspluralismus, in: Rechtshistorisches Journal 15 (1996), 255; FRIEDRICH MUULLER/RALPH CHRISTENSEN, Juristische Methodik. Bd. Il: Europarecht. Berlin 2003. 
stimmte Möglichkeiten betrachtet werden, nämlich die Medien des Rechts, und hierbei wiederum nur ein unbestimmtes Medium, das Bild.

\section{Dynamik zwischen Barbarei und Blabla}

Es stellt sich die Frage nach der Besonderheit einer Medientheorie des Rechts und den Besonderheiten der damit verbundenen Grenzziehungen. Grundsätzlich ist anzunehmen, dass Formen und Inhalte der Rechtskommunikation konstitutiv mit den Medien der Rechtskommunikation verknüpft sind, da Verbreitungsmedien zugleich als Speicher der Kommunikation fungieren. Sie sind Archive, die "die Gedächtnisformen des Rechts strukturieren und damit sowohl die Bedingungen der wiederholten Verwendbarkeit rechtlichen Wissens konditionieren, als auch den Grad der Neigung, tradierte Wissensbestände zu variieren." 12

Unabhängig davon, welche Möglichkeit der Grenzziehung zwischen Recht und Nichtrecht heute gewählt wird, ist doch insgesamt eine erhebliche Temporalisierung und Mobilisierung der Rechtsvorstellungen zu beobachten. ${ }^{13}$ Gerade dies macht die Sprache des Rechts als theoretischen Gegenstand so attraktiv. Sprache ist eben Gerede, das sich in der Unheimlichkeit der Schwebe hält. ${ }^{14}$ Zugleich geraten die Beziehungen des Rechts zur Kultur und zur prekären Stabilität ihrer Dynamik ins Blickfeld - zu anderen Zeiten und an anderen Orten wird anders entschieden. ${ }^{15}$ Die Sprachen des Rechts haben ein ähnliches Problem wie die Kulturen des Rechts. Sie stehen unter Vergleichszwängen. Man muss das Recht auch und man kann es nur über seine eigenen Grenzen hinweg vergleichen. Und das heißt: Womit? Mit anderem Recht? Mit etwas anderem als dem Recht? Mit ungriechischer Barbarei? Mit theoretischem Blabla? Und ist das nicht das Gleiche?

12 ThOMAS VeSTING, Die Medien des Rechts. Sprache-Schrift-Buchdruck-Hypertext, http:// www. jura.uni-frankfurt.de/vesting/Medien-des-Rechts.html v. 20.8.2004.

$13 \mathrm{Vgl}$. anhand des Beispiels europäischer Rundfunkregulierung THOMAS VESTING, Die Staatsrechtslehre und die Veränderung ihres Gegenstandes: Konsequenzen von Europäisierung und Internationalisierung, in: VVDStRL 63 (2004), 41-68, 49 ff.; GUNTHER TEUBNER, Globale Zivilverfassungen. Alternativen zur staatszentrierten Verfassungstheorie, in: MARCELO NEVES/RÜDIGER VOIGT (Hrsg.), Zur Staatstheorie Niklas Luhmanns. Baden-Baden 2003.

14 MARTIN HEIDEGGER, Sein und Zeit (1927). Tübingen 1993, 170.

15 Siehe NiKLAS LUHMANN, Kultur als historischer Begriff, in: Gesellschaftsstruktur und Semantik. Bd. 4. Frankfurt am Main 1995, 31; DIRK BAECKER, Wozu Kultur? Berlin 2000 44; Heiner MüHLManN, Die Natur der Kulturen. Wien 1996, 16 ff. 
Die Attraktivität der Vorstellung, dass Recht eine Sprache sei, trägt sowohl den Temporalisierungen und Mobilisierungen der Grenzvorstellung Rechnung als auch den Vergleichszwängen von Kulturen. Es verschärft sie aber $z \mathfrak{u}$ Übersetzungszwängen. ${ }^{16}$ Die Frage, was Recht sei, wird unter dem titelgebenden "Sprachen des Rechts" umformuliert zu: Wann ist Recht? ${ }^{17}$ Die Antwort lautet vielleicht lapidar: Wenn darüber kommuniziert wird und das heißt, wenn Kommunikation mit der Leitunterscheidung zwischen Recht/Unrecht (Luhmann) bzw. Recht/ Nichtrecht (Wiethölter) und mit der Suche nach Vergleich und Übersetzung stattfindet. ${ }^{18}$ Die Referenzstruktur des Rechts, für deren Beschreibung so unterschiedliche Modelle wie Geschichte, Gedächtnis, Rhetorik oder Evolution verwendet werden, ist in allen diesen Modellen durch eine Logik der Unterscheidung und Selektion gekennzeichnet. Wenn man mit dem Fokus auf die Unterscheidungsdynamik der Kommunikation heute auch zwischen die oben erwähnten Fronten des 19. Jahrhunderts gerät, so sind diese Fronten damit natürlich noch nicht überwunden. Wie auch, wenn alles dazu dient, Recht von Unrecht und von Nichtrecht zu unterscheiden, Fronten also aufzubauen und dabei verbindlich zu bleiben. Und das heißt, dass sich auch in der Art und Weise, wie Kommunikation und Medien des Rechts beschrieben werden, vermutlich materialistische oder idealistische Erbschaften finden. So flüchtig und beweglich man das Recht in der Kommunikation noch schildern mag: Gesetz ist nicht Gesetz, aber nur Recht ist Recht. Reflektionen über die Sprache des Rechts haben also damit umzugehen, dass sich das Recht durch die Einheit von Stasis und Wandel hält und dass damit Einheit und Differenz des Rechtes korrelativ bleiben. ${ }^{19}$ Das ist die Paradoxie der Identität bei gleichzeitiger Veränderung (Fögen).

Paradoxien zwingen nicht nur dazu, kenntlich zu machen, was diesseits und jenseits des Rahmens steht, der durch die Paradoxie gebrochen wird. Sie erleichtern diese Markierung auch erheblich durch die klärende Kraft des Rahmens, den sie setzen. Im Kontext der genannten Paradoxie lassen sich (heuristisch-vereinfachend) zwei rechtswissenschaftliche Pole kennzeichnen, zwischen denen die Beschreibung, was ein Kommunikationssystem ist, changiert, nämlich zwischen der Kommunikation, die stattfindet, und den Medien, mit und in denen sie statt-

16 LUHMANN, Recht (Fn. 9), 102.

17 In Abwandlung zu NELSON GOODMAN, Wann ist Kunst?, in: DERS., Weisen der Welterzeugung. Frankfurt am Main 1990, 76

18 TEUBNER, Rechtsparadoxien (Fn. 6), $33 \mathrm{f}$

${ }^{19}$ Zur Einheit von Stasis und Wandel als Teil der Evolution des Rechtes siehe: MARC AMSTUTZ, Evolutorisches Wirtschaftsrecht. Vorstudien zum Recht und seiner Methode in den Diskurskollisionen der Marktgesellschaft. Baden-Baden 2001; DERS., Rechtsgeschichte als Evolutionstheorie, in: Rechtsgeschichte 1 (2002), 26. 
findet. ${ }^{20}$ Auf der einen Seite der Paradoxie lässt sich die Vermittlungsleistung des Kommunikationssystems - seine Referenzstruktur - daher über die Formationskraft der Trägermedien (Sprache-Schrift-Bild-Buchdruck oder Hypertext) beobachten. Auf der anderen Seite lässt sie sich auch über die Vermittlungsleistung in der Codierungskraft laufender Umcodierung in symbolisch generalisierten Kommunikationsmedien beobachten. ${ }^{21}$ Jochen Schulte-Sassen hat hierzu vorgeschlagen, von harten und schwachen Medientheorien zu sprechen, um herauszustellen, wie in den theoretischen Beschreibungen jeweils die Materialität der Zeichenträger, die Idealität von Bedeutungszuschreibungen und die damit verbundenen strukturierenden Kräfte bewertet werden. ${ }^{22}$ Die erwähnten (harten und weichen) medientheoretischen Pole schaffen für die Beschreibung des Rechts Beobachtungsfelder, innerhalb derer Wandel und Stasis des Rechts unterschiedlich dargestellt und die Materialität und Idealität der Rechtskommunikation unterschiedlich bewertet werden können. Einerseits lassen sich nun Rechtstheorien danach analysieren, ob sie einen harten oder schwachen Medienbegriff verwenden, und diese Analyse lässt sich wohl auch auf juristisch geläufigere Unterscheidungen zwischen Begriff und Interesse, Formalität und Inhaltlichkeit oder Subjektivität und Objektivität des Rechts beziehen. Mit dieser Form der Übersetzung glückt sicherlich eine Aktualisierung der Rechtstheorie im Sinne einer Anpassung der Rechtstheorie an die Entwicklungen in den Nachbarwissenschaften. Entscheidend ist aber etwas anderes, eine weitere Form der Aktualität und zwar der Umstand, dass in einem Bezug zu den Medien der Kommunikation die juristisch geläufigen Unterscheidungen selbst in einem Status laufender Aktualität gehalten werden. Auch wenn man sich im Kampf ums Recht für Begriff oder Interesse entscheiden kann: im nächsten Moment muss man sich wieder entscheiden und die Medientheorien liefern hier einen beunruhigenden Störfaktor. Deutlich wird dies, wenn man versucht, eine juristische Entscheidung medientheoretisch zu analysieren. Die Dynamik des Rechts ist getaktet, und daher tendiert der Prozess juristischer Umcodierung immer wieder zur Form und von ihr weg. Juristische Kom-

\footnotetext{
20 Dabei stehen Medien und Kommunikation selbst wieder in einem Spannungsverhältnis zwischen dem materialen Gefüge der Zeichenträger und dem kontingenten Prozess der Bedeutungsauswahl. Vgl. zum Beispiel die Beschreibung bei MANFRED FARLER, Was ist Kommunikation? München 1997, $22 \mathrm{f}$.

${ }^{21} \mathrm{Zu}$ symbolisch generalisierten Kommunikationsmedien: LuHMANN, Die Gesellschaft der Gesellschaft (Fn. 4), 316 ff., zur Rationalität der Kommunikation u. a. JURGEN HABERMAS, Faktizität und Geltung. Beiträge zur Diskurstheorie des Rechts. Frankfurt am Main 1994, 15 ff., 151 ff., $187 \mathrm{ff}$.

22 JOCHEN SCHULTE-SASSEN, Medien (Fn. 5), 1.
} 
munikation wird also z. B. in $\operatorname{Akten}^{23}$ (z. B. BvR 1190/90; 1 BvR 2173/93; 1 BvR 433/96), ${ }^{24}$ in Druckerschwärze, Schallwellen, Begriffen („Nötigung") ${ }^{25}$ in einer Grammatologie ${ }^{26}$ oder einer Grammatik des Begründung ${ }^{27}$ festgehalten - aber nur für bestimmte Taktschritte, die dann z. B. als Datum einer Bundesverfassungsgerichtsentscheidung (24.10.2001) oder als Erscheinungsdatum einer Entscheidungssammlung bzW. einer Zeitschrift (NJW 2002) oder auch als evolutionäre Errungenschaft einer Kontingenzformel ("Nötigung") Anstoß für Weiteres und weitere Taktschritte geben.28 Weil die getaktete Dynamik juristischer Umcodierung immer wieder zur Form und von ihr weg tendiert, kann jeder dieser Momente und seiner Folgemomente als Medium oder als Form beobachtet werden. Jeder dieser Momente kann Aufmerksamkeit binden und so als Besonderes in seiner Relation zum Allgemeinen beobachtet werden. Was das Allgemeine des Besonderen et vice versa aber ist, wird davon abhängen, was man beobachtet, ob man es als Medium oder Form beobachtet und wie dabei Inhalt und System in den Hintergrund treten. Jedes Datum in der Kommunikation kann dabei innerhalb und außerhalb der Kommunikation stehen, in dem Sinne, dass es als Kommunikationsmedium auf die Einheitsbildung des Rechts bezogen wird oder indem es als verselbstständigte Form außerhalb von Kontinuitäten beobachtet wird. ${ }^{29}$

So kann man zum Beispiel medientheoretische Analysen auf die Beziehung zwischen öffentlichem Recht und Staat und die dazwischen

23 Dazu: CORNelia ViSManN, Akten. Medientechnik und Recht. Frankfurt am Main 2000.

${ }^{24}$ Grundlage für: BVerfG, Urteil v. 24.10 .01 (Sitzblockade II) = NJW 2002, 1031.

${ }^{25}$ Siehe dazu zuletzt: BVerfG, Urteil v. 24.10 .01 (Sitzblockade III) = NIW 2002, 1031.

26 JACQUES DERRIDA, Grammatologie. Frankfurt am Main 1983.

27 Dazu: MANFRED BIERWISCH, Recht linguistisch gesehen, in: GÜNTHER GREWENDORF (Hrsg.), Rechtskultur als Sprachkultur. Zur forensischen Funktion der Sprachanalyse. Frankfurt am Main 1992, 42

${ }^{28}$ Luhmann beschreibt dies als die Zirkulation von Medien, vgl. LUHMANN, Die Gesellschaft der Gesellschaft (Fn. 4), 198-202.

$29 \mathrm{Vgl}$. als Beispiel den Streit um die Kommentierung der Menschenwürde. Jeder Kommentar zu diesem Streit berührt das Problem der Unberührbarkeit der Menschenwürde und ist in diesem Sinne als Medium und als Form beobachtbar und verteidig- und angreifbar; siehe dazu: ERNST-WOLFGANG BÖCKENFÖRDE, Die Menschenwürde war unantastbar, in: FAZ vom 3. September 2003.; ROBERT LEICHT, Wahret die Anfänge, in: Die Zeit 38/2003; DERS., Tasten nach der Würde. Geschichtsvergessen - Der neue Kommentar zum Grundgesetz, in: Tagesspiegel vom 15. September 2003; UWE JUSTUS WENZEL, Menschenwürde und Menschenbild. Über die Relativität des Absoluten, in: NZZ vom 15.11.2003; CHRISTINE FRANZIUS, Der ausgefallene Juristenstreit http:/ / hsozkult.geschichte.hu-berlin.de/forum/id=388\&type=diskussionen; als Unterschied zwischen loser und fester Kopplung beschrieben bei LUHMANN, Die Gesellschaft der Gesellschaft (Fn. 4), 198 ff, und bei BAECKER, Kommunikation im Medium der Information (Fn. 4), 111 
laufende oszillierende Grundbewegung zwischen der Systembildung des öffentlichen Rechts und der Entscheidungsformbindung in Verfassungsurkunden und Gesetzes- oder Urteilstexten beziehen. Sie lässt sich auf die Verselbstständigungsdynamiken im Europarecht, z. B. der europäischen Rundfunkregulierung, im Völkerrecht, z. B. im WTORecht und seinem Streitbeilegungsverfahren, in den privat-öffentlichen Regulierungsnetzwerken und nicht zuletzt in der Global Bukowina der Software-Standards beziehen. Die Kritik auf alle möglichen der Verselbstständigungsdynamiken des Rechts zu beziehen heißt, sie auf die Probleme der Form zu beziehen. Die Verselbstständigungsdynamiken sind dabei aber nur schwer mit Formbegriffen wie Offener Staat, Offener Interpretationskreis oder Konstitution zu beschreiben. ${ }^{30}$ Der Kommunikationsdynamik ist eigen, dass in ihr zwar die Dialektik von morphe bzw. Form und dynamis wirksam wird, dass sie zugleich aber historisch gebunden wird an eine Geschichte des Rechtsbegriffs und so die historische, strikte Kopplung jene Dialektik zur Standeskunst einer veraltenden Rechtswissenschaft zu zähmen droht. Was passiert, wenn Veraltung droht? "Einst" hat die Rechtswissenschaft auf die Probleme der Form unter den Bedingungen der Dynamik mit der Metaphorisierung und Fiktionalisierung ihres Gegenstandes reagiert. ${ }^{31}$ Seitdem die Relation von Form und Inhalt in eine Begleitsemantik der modernen Kunst abgedrängt wurde, und seitdem dort innerhalb der analogen semiotischen Relation von Zeichen und Referenz das Zeichen nur noch zum Ausdruck bringt, dass es keine Zeichen gibt ${ }^{32}$ (Ceci n'est pas une pipe), leiten diese Metaphorisierungs- und Fiktionalisierungsbestrebungen aber nicht wirklich weiter, weil sich mit ihnen nicht der eigenartige Status der Metapher zwischen Bild und Begriff klären lässt. Jetzt - wie zum Beispiel im Europa des Jahres 2004 - kann man eben auf den Hinweis, das Verträge keine Verfassung sind nicht einfach mit der Replik antworten, dann sei Verfassung eben eine Metapher. Auch auf den Hinweis, dass Europa kein Staat sei, kann man jetzt nicht einfach antworten, dann sei Staat eben eine Metapher. Natürlich war einst schon immer einst und jetzt schon immer jetzt. Das heißt aber nur, dass Probleme der Form schon immer Probleme der Zeit waren und nicht, dass die Probleme der Form verschwinden. Auch in der Umstellung auf die Dynamik der Kommunikationsbedingungen bleiben mit ihren Formu-

${ }^{30} \mathrm{Vgl}$. VESTING, Staatsrechtslehre und Veränderung (Fn. 12), 64 ff.

${ }^{31}$ Zum Beispiel: Hugo Grotius, De jure belli ac pacis libri tres (1625). Tübingen 1950, mit der Formel des "etsi deus non daretur".

32 NiKLaS LuHMANN, Die Kunst der Gesellschaft. Frankfurt am Main 1995, 110; Ders., Zeichen als Form, in: DIRK BAECKER (Hrsg.), Probleme der Form. Frankfurt am Main $1993,46$. 
lierungsschritten Identität und Differenz des Rechts korrelativ; es ist nur sinnvoller, von Formbegriffen auf Medienbegriffe umzustellen, um weiter Herr der Differenzierungsoperationen zu bleiben. Innerhalb der Kontingenz mehrfacher Selektionsbedingungen der Kommunikation ist es dabei weder sinnvoll, sich auf die Seite der Form, noch auf die Seite des Inhalts zu schlagen. ${ }^{33}$ Beide Entscheidungen tragen den Vergleichsund Übersetzungszwängen über Grenzen hinweg nicht hinreichend Rechnung.

So einfach uns die Paradoxie der Identität bei gleichzeitiger Veränderung vielleicht theoretische Positionen markieren lässt, man wird zugleich hineingezogen in eine unendliche Anzahl von Entscheidungen: worauf man sein Modell der Einheitsbildung beziehen möchte und worauf nicht, wie man mit Identität und Differenz des Rechts umgehen möchte, und was von dem, was man vom Recht kennt, fortführen und was aufgeben möchte. Zu einer Paralyse des Rechtsdenkens führt die Medientheorie (hoffentlich) nicht. Dies gilt schon deswegen, weil die Praxis der Rechtsentscheidungen von Medien- und Kommunikationstheorien keine Kenntnis nimmt. Aber auch auf einer theoretischen Ebene paralysiert diese Unendlichkeit der Entscheidungsmöglichkeiten nicht. Es handelt sich eher um das Kollabieren der Unterscheidung, das in der Systemtheorie als Wiedereinführung der Unterscheidung in das Unterschiedene (re-entry) beschrieben wird und das früher für die Romantiker den Zauber des Unverständlichen in der laufenden Reflexion. entfaltete. ${ }^{34}$ Die Unverständlichkeit, von der die Romantiker sprachen, macht die Kommunikation nicht unmöglich, sondern eröffnet ihr ihren Spielraum. Dirk Baecker hat dazu angemerkt, dass die Medientheorien vor dem Hintergrund des Interesses an der Differenz und als Beschreibung der Differenz gelesen werden müssen, und er nennt hier vor allem die Arbeiten McLuhans und Derridas über die Schrift als Beispiele. ${ }^{35}$ Nach Baecker ist für die Medien- und Kommunikationstheorien wesentlich, dass immer eine Differenz (zwischen Form/Inhalt; Figur/ Grund; Anwesendem/Abwesendem) mitgedacht und theoretisch formuliert wird. "In diesem Sinne“, so Baecker, "arbeiten Medientheorien an der Bestimmung des dritten Wertes der dreiwertigen Zweiseitenform der Kommunikation" um mit der Temporalisierung des Rechts und seinen Formgebungen und so mit seinem dynamischen Verhältnis

\footnotetext{
${ }^{33}$ Siehe zu den Selektionsbedingungen der Kommunikation (im Hinblick auf Information-Mitteilung-Verstehen) LUHMANN, Die Gesellschaft der Gesellschaft (Fn. 4), 190-202.

${ }^{34}$ Dazu: FRIEDRICH SCHLEGEL, Über die Unverständlichkeit (1800), in: Kritische FriedrichSchlegel-Ausgabe. Bd. 2. München 1967, 363.

35 BAECKER, Kommunikation (Fn. 4), 423.
} 
zum Nicht-Recht umzugehen. ${ }^{36}$ Auch wenn sich also mit den Medientheorien theoretische Erbschaften aus dem 19. Jahrhundert etwa in der Art der Unterscheidung nach Materialität und Idealität des Rechts aufspüren lassen, so erlaubt die Vorstellung des Mediums eben nicht, sich auf eine Seite dieser Fronten zu schlagen. ${ }^{37}$ Die Besonderheit der Medientheorien liegt in dieser Unmöglichkeit und in der damit verbundenen Möglichkeit, den Blick auf die Operationen der Differenzierung selbst zu lenken. Sollte irgendwann in diesem Beitrag noch einmal von Bildern die Rede sein, so kann dies nur im Sinne der medientheoretischen Besonderheit gemeint sein.

\section{Bilderflu(ch)t}

Es gibt zahlreiche Diagnosen zum Verschwinden der Bilder aus dem Recht, zur Ikonophobie des modernen Rechts, und zahlreiche Diagnosen zur visuellen Zeitenwende, zur Bilderflut (Iconic Turn) der jüngeren Zeit. ${ }^{38}$ Die Grenzbestimmungen des Rechts sind dabei eng mit den Grenzbestimmungen des Bildes verbunden: was Recht ist, wird in Abhängigkeit dazu bestimmt, was (k)ein Bild ist. Hierzu sind drei zentrale Thesen zu referieren.

1. These: Das nachhumanistische Methodenideal der Rechtswissenschaft ist bilderfeindlich geworden.

Vor allem an dem Wandel der Rolle der Rhetorik im Recht lässt sich die moderne Bilderfeindlichkeit des Rechts ablesen. Die Symptome für den Wandel sind zum Beispiel das Verschwinden von Bildern aus juristischen Lehrbüchern, der Verzicht auf Illustrationen in Monographien, Gesetzestexten und Verträgen und die Marginalisierung der Rhetorik im juristischen Unterricht. ${ }^{39}$ Hinzu kommt, dass die juristische Rhetorik in einigen rechtswissenschaftlichen Arbeiten erheblich verengt nur noch als hermeneutische Technik, das heißt als Mittel, Schriften auszulegen, dargestellt wird und zugleich in einer Recht und Text zusammenführenden Rückprojektion behauptet wird, die antike Rhetorik sei

\footnotetext{
36 BAECKER, Kommunikation (Fn. 4), 423.

37 Dazu auch ebd., 122.

$38 \mathrm{Vgl}$. nur die Beiträge in Fn. 1.

39 Vgl. u. a. die Darstellung bei GERT UEDING/BERND STEINBRINK, Grundriss der Rhetorik. Geschichte - Technik-Methode. Stuttgart 1994, 142-154 sowie den Beitrag von KLAUS F. RÖHL, Bilder in gedruckten Rechtsbüchern, im vorliegenden Band 267-348.
} 
vor allem Gerichtsrhetorik gewesen. ${ }^{40}$ Damit zusammenhängend wird auch vom Ende der Rhetorik bzw. deren Ablösung durch die Hermeneutik gesprochen. ${ }^{41}$ Andere, die sich für eine rhetorische Schulung im Recht auch heute stark machen, unterstellen für die Rhetorik zugleich eine klare Unterscheidbarkeit zwischen Argument und Figur, zwischen eigentlichem und uneigentlichem Sprechen. ${ }^{42}$ Insbesondere in der neuen Rhetorik der Argumentationstheorien bemühen sich die Autoren darum, analytische Instrumente zu finden, um Argumente von Figuren zu unterscheiden und um so die Rationalität des Rechts über die Unterscheidung von gültiger und ungültiger Kommunikation zu sichern. ${ }^{43}$ Insoweit wird die Entwicklung der neuzeitlichen Rechtsmethode als Befreiung aus einer rhetorischen Verstrickung und Errungenschaft kommunikativer Eigenrationalität erzählt, deren Gewinn und Bedingung in einer prägnanten Unterscheidbarkeit rechtshistorisch zwischen Fakt und Fiktion und argumentationstheoretisch zwischen Argument und Figur und damit in einer semantischen und syntaktischen Verschärfung (claritas) der Rechtssprache liegt. Diese erfüllt auf kommunikativer Basis eine Funktion des Rechts, nämlich Verhaltenserwartungen zu stabilisieren und für beide Seiten kontrollierbar zu machen. ${ }^{44}$

${ }^{40}$ Siehe z. B. JAN SCHRÖDER, Rhetorik und juristische Hermeneutik in der frühen Neuzeit, in: Richard Helmholz/Paul Mikat/Michael StOlleis (Hrsg.), Grundlagen des Rechts. Paderborn 2000, 677.

${ }^{41}$ SCHRODER, Rhetorik und juristische Hermeneutik (Fn. 40), 678.

42 Katharina von Schlieffen verwendet ein Instrument, nach dem sich die Anzahl der rhetorischen Figuren im Verhältnis zu den Argumenten unterscheiden lassen. Auf diese Weise soll sich die Dichte des tropischen Sprechens in Gerichtsurteilen zwischen niedrig (rechtsgültige Argumentation) und hoch (figürliche Rede) bestimmen lassen. Vgl. zuletzt KATHARINA GRÄFIN VON SCHLIEFFEN, Rhetorik, in: VOLKER ROMERMANN/ CHRISTOPH PAULUS (Hrsg.), Schliisselqualifikationen für Jurastudium, Examen und Beruf. München 2003, 192 und KATHARINA GRÄFIN VON SCHLIEFFEN, Zur topisch-pathetischen Ordnung juristischen Denkens - Resultate empirischer Rhetorikforschung, in: KENT D. LERCH (Hrsg.), Recht verhandeln. Argumentieren, Begriinden und Entscheiden im Diskurs des Rechts. Berlin 2005, 403-446.

${ }^{43}$ Stellvertretend: ROBERT ALEXY, Theorie der juristischen Argumentation. Die Theorie des rationalen Diskurses als Theorie der juristischen Begründung (1978). Frankfurt am Main 1983.

${ }^{44}$ Symptomatisch auch, wenn im Lexikon über Juristen ein Eintrag über Machiavelli fehlt. Erst, wenn Klarheit als Vorzug der Rechtsmedien beschrieben wird und das Bild als unklares Medium dem Kommunikationsmedium Macht zugeordnet ist, erscheint die Lehre Machiavellis vom arcanum imperii als unjuristisch, vgl. dazu auch: ELISABETH VON HAGENOW, Bildniskommentare, Allegorisch gerahmte Herrscherbildnisse in der Graphik des Barock. Hildesheim 1999, 136 ff.; zum abwesenden Machiavelli siehe MiCHAEl StOlleis (Hrsg.), Juristen. Ein biographisches Lexikon, München 1995, 394 und MiCHAEL STOLlEIS, Staat und Staatsräson in der frïhen Neuzeit, Frankfurt am Main 1990, 21-37; zur Vorstellung semantischer und syntaktischer Verschärfung siehe RÖHL, Bilder in gedruckten Rechtsbüchern (Fn. 39). 
Bilderfeindlichkeit erscheint mit dieser These als stabilisierende Kommunikationsbedingung.

\section{These: Der moderne Rechtsstaat war ikonophob.}

Die zweite These ist eng mit der ersten verbunden, bezieht sich aber nicht bloß auf das Methodenideal der Rechtswissenschaft, sondern auf die Formulierungs-, Formierungs- und Konstitutionalisierungskräfte des Rechtssystems gegenüber anderen gesellschaftlichen Teilbereichen selbst und nicht nur auf die Unterscheidung von Begriff und Sprachbild sondern auch auf die Unterscheidung von visuellen Bildern (z. B. Tafelbild, Fresko, Druckgraphik) einerseits und Sprache, Schriftlichkeit, Buchdruck und Rede andererseits. Die Ikonophobie des modernen Rechtsstaates scheint eine Bedingung seiner Autonomie zu sein, weil sie Bedingung der Abgrenzung gegenüber Kunst, Moral und Politik ist. Symptom hierfür ist das Verstummen der Ikonologie/Ikonographie im Recht. Latent sind auch heute Rechtssätze und Rechtsprinzipien illustrierbar ${ }^{45}$ und es finden sich auch heute genug Bilder, die man mit rechtlichen Kategorien zum Sprechen bringen kann. ${ }^{46}$ Die Ikonologie/Ikonographie des Rechts ist also allenfalls verstummt, nicht verschwunden. Ein erheblicher Wandel gegenüber den Kommunikationsbedingungen des Rechts zur Zeit der höfischen Beredsamkeit steht aber nicht in Frage. ${ }^{47}$ Die bis heute wirkungsmächtigste Schilderung dieses Wandels hat Hegel in der Einleitung seiner Grundlinien der Philosophie des Rechts mit der Beschreibung der Entstehung des bürgerlichen Rechtsstaates und dem Ende der Kunst geliefert. ${ }^{48}$ Dort heißt es im Sinne der oben beschriebenen Alterungsprozesse des Rechts:

"Als der Gedanke der Welt erscheint sie [die Philosophie des Rechts] erst in der Zeit, nachdem die Wirklichkeit ihren Bildungsprozeß vollendet und sich fertig gemacht hat. Dies, was der Begriff lehrt, zeigt notwendig ebenso die

${ }^{45}$ Zum Beispiel der Beschluss des Deutschen Bundestages zur Errichtung eines Mahnmals für die ermordeten Juden Europas vom 6. Juli 1999 (BT Drucks. 14/1238): „Der Bundestag folgte damit der vom Ausschuss einstimmig bekundeten Absicht, mit dem Denkmal die ermordeten Opfer zu ehren, die ,Erinnerung an ein unvorstellbares Ereignis der deutschen Geschichte' wachzuhalten und ,alle künftigen Generationen' zu mahnen, nie wieder die Menschenrechte anzutasten, den demokratischen Rechtsstaat zu verteidigen, die Gleichheit der Menschen vor dem Gesetz zu wahren und jeder Diktatur und Gewaltherrschaft zu widerstehen" (Pressemitteilung Juli 1999).

${ }^{46}$ Zum Beispiel JÜrGen HABERMAS, Was bedeutet der Denkmalsturz? Verschließen wir nicht die Augen vor der Revolution der Weltordnung: Die normative Autorität Amerikas liegt in Trümmern, in: $F A Z$ vom 17. April 2003, 33.

$47 \mathrm{Vgl}$. ADAM MÜLLER, Zwölf Reden über die Beredsamkeit und deren Verfall in Deutschland (1812). Recklinghausen 2002; WOLFGANG BRASSAT, Das Historienbild im Zeitalter der Eloquenz. Berlin 2003.

${ }^{48}$ Dazu auch Fabian SteinhaUer, Princeps iustitiae advigilans. Zur Bildgeschichte des Auges und zur Poetik des Staates, in: Der Staat 2004, 628-636. 
Geschichte, daß erst in der Reife der Wirklichkeit das Ideale dem Realen gegenüber erscheint und jenes sich dieselbe Welt, in ihrer Substanz erfaßt, in Gestalt eines intellektuellen Reichs erbaut. Wenn die Philosophie ihr Grau und Grau malt, dann ist eine Gestalt des Lebens alt geworden, und mit Grau und Grau läßt sie sich nicht verjüngen, sondern nur erkennen ... ${ }^{\prime 49}$

Mit der Errungenschaft des modernen Rechts ist die Gesellschaft in ein Stadium der Nüchternheit eingetreten, in der der Spielraum für die Poesie (Wyss), für Kunst und Bilder schwindet - der Anfang dieses Rechts ist das Ende der Kunst. ${ }^{50}$

\section{These: Das Recht gerät unter medialen Anpassungsdruck.}

Diese letzte These betrifft ebenfalls die Kollision des Rechts mit anderen Rationalitätsbedingungen der Gesellschaft, versucht diese Kollision aber nicht dadurch aufzulösen, dass die Autonomie gesellschaftlicher Teilbereiche immer schon vorausgesetzt wird, sondern indem Kollisionsregeln für das Verhältnis zwischen Recht, Kunst und der zeitgenössischen Darstellungspraxis gesucht werden..$^{51}$ Schon $z u$ Beginn des 20. Jahrhunderts erfolgt der Hinweis auf den medialen Anpassungsdruck des Rechts: „Es wäre ein gefährliches Unterfangen, wenn sich Personen mit einer ausschließlich juristischen Ausbildung zu endgültigen Richtern über den Wert von bildlichen Darstellungen aufschwingen würden", merkt Oliver Wendell Holmes 1903 in der Entscheidung Bleistein gegen Donaldson an. ${ }^{52}$ Was hier als Konflikt zwischen persönlicher Kompetenz des Richters (denn die wird schließlich vorausgesetzt) und Komplexität ästhetischer Fragestellungen thematisiert wird, wird zum Problem der Eigenrationalität des Rechts, wenn es selbst schon wie z. B. mit dem Medienrecht, der Einräumung von Kommunikationsfreiheiten und internen Kollisionsregeln $\mathrm{zu}$ anderen Rechten wie z. B. dem allgemeinen Persönlichkeitsrecht Kategorien des Bilderstreites ins Recht implementiert. Unabhängig von der modernen Ausdifferenzierung des Rechts gegenüber anderen gesellschaftlichen Teilbereichen wie der Kunst ist der Bilderstreit auch erst im modernen Recht verrechtlicht worden, in Deutschland mit dem KUG von 1907.

${ }^{49}$ GeORG FRIedrich Wilhelm Hegel, Grundlinien der Philosophie des Rechts. Frankfurt am Main 1986, 28.

${ }^{50}$ Der "Staat mit seinen Rechten und Verpflichtungen erscheint hier [bei Hegel] als das wirkliche Gefäß, das allein die Wahrheit in ihrer philosophischen Strenge und Folgerichtigkeit fassen konnte. Kunst wog zu leicht für das Leben.", so BEAT WYSS, Trauer der Vollendung. Zur Geburt der Kulturkritik. Köln 1997.

5] Zum Beispiel KarL-HeINZ LADEuR, Die Anpassung des Medienrechts an die Unterhaltungsöffentlichkeit, in: NJW 2004, 393-398.

52 Zitiert nach COSTAS DOUZINAS, Die Legalität des Bildes, in: SCHWARTE/Wulf, Körper und Recht (Fn. 1), 147. 
Dabei stellt sich die Frage, ob die Konzepte der Kommunikationsfreiheit des Rechts auf der Basis von gedruckter Schrift entwickelt wurden und ob sie angesichts der "Medienentwicklung im post-gutenbergschen Zeitalter überholt" sind. ${ }^{53}$ Die Kollision betrifft auch hier zwei Ebenen, die man analytisch auseinander halten kann und die gleichwohl zusammenhängen, wenn man Medientheorie in gesellschaftstheoretischer Perspektive betrachtet. Sowohl auf der basal-medialen Ebene des Trägermedienwechsels von Buchdruck zum elektronischen Hypertext als auch auf der gesellschaftlichen Ebene der Kollision eines Prinzips der repräsentativen Demokratie gegenüber den gewandelten Bedingungen der Massenmedien sind Konflikte zu erwarten.

\section{Paragone der Rechtsmedien}

\section{Die Entstehung von Leitmedien}

Der Begriff Paragone ist dem italienischen Humanismus entlehnt, er bezeichnete dort den Wettstreit verschiedener Künste (Architektur, Malerei, Bildhauerei) um den Status einer freien Dichtkunst im Sinne der artes liberales. Zumindest mit den zwei erstgenannten Thesen gerät man in einen Paragone mit umgekehrten Vorzeichen, indem man um Leitmedien des Rechts zur Sicherung seiner Autonomie streitet. Eine Aussage über die historische Stichhaltigkeit oder Fragwürdigkeit dieser Thesen $\mathrm{zu}$ machen, ist schon deswegen schwierig, weil erst einmal geklärt werden müsste, ob und was das denn für ein Recht war, aus dem die Bilder verschwunden sind, und ob und was das denn für ein Nichtrecht ist, in das sie verdrängt wurden oder zu dem sie geflüchtet sind. Es ist zu klären, was das denn für Bilder waren, die da verschwanden, und was das denn für Nichtbilder sind, die im Recht zurückgeblieben sind. Ein Unterfangen, das nicht nur im Hinblick auf die oben geschilderte Formbindungsdynamik der Unterscheidung, sondern auch im Hinblick auf die Weite und Unschärfe des Bildbegriffes nicht gerade einfach erscheint. Der Begriff des Bildes umfasst auch in der zeitgenössischen Literatur (vgl. Fn. 1) von eicon, eidolon über imago, effigies, simulacrum, pictura bis hin zu Bildnis, Image, Anschauung, Vorstellungskraft, Symbol, Metapher, Kunst, Vergleich und zum logischen Bild im Sinne Wittgensteins so ziemlich alles, was im Zusammenhang mit der Repräsentation von Sinn zweifelhaft ist. Ich möchte mich daher damit begnügen, den Blick auf den Zusammenhang der Differenzierung von

53 FRANKENBERG/NieSEN, Bilderverbot (Fn. 1), 5. 
Recht/Nichtrecht und Bild/Nichtbild zu lenken. Dabei gibt es zugleich einen Zusammenhang zwischen dem, wie das Bild als basales Trägerund Verbreitungsmedium bestimmt wird, und der Zuordnung von Bildern zu Kommunikationsmedien wie Kunst, Recht oder Macht. Dieser Zuordnung von Medien als basalen Träger- und Verbreitungsmedien $\mathrm{zu}$ bestimmten Kommunikationsmedien führt in den soziologischen Medientheorien zu der Vorstellung von Leitmedien in gesellschaftlichen Teilbereichen. Dabei werden wiederum den basalen Medien unterschiedliche kommunikationsstabilisierende Wirkungen zugeschrieben, und sie entwickeln eine Mediensemantik zwischen Freiheit und Zwang, von der die früheren Medientheorien eigentlich absehen wollten, als sie mit dem Slogan "Das Medium ist die Botschaft" den Vorrang formaler Medientechnik gegenüber allen Semantiken propagierten. Ein Beispiel für die Zuordnung von Trägermedien zu Kommunikationsmedien ist die Zuordnung des Trägermediums Sprache/Buchdruck zum Kommunikationsmedium Recht und die Zuordnung des Trägermediums Bild zum Kommunikationsmedium Macht oder Kunst. ${ }^{54}$ Für die Vorstellung unterschiedlicher kommunikationsstabilisierender Wirkungen steht etwa der Satz: „Ein Bild sagt mehr als tausend Worte." Die Tradition des Paragone ist selbst wiederum Beispiel für die Mediensemantik zwischen Freiheit und Zwang. ${ }^{55}$

Im Recht ist das Leitmedium die Sprache, genauer gesagt: die Schriftsprache unter den Bedingungen des Buchdruckes, nicht allein diese Schriftenreihe zeigt das. Das Bild ist in der durch Gadamer geprägten Hermeneutik, ${ }^{56}$ in der durch die analytische Philosophie geprägten Argumentationstheorie ${ }^{57}$ und sogar in der soziologischen Medientheorie Luhmanns das Leitmedium der Kunst, oder es wird dem Kommunikationsmedium Macht zugeordnet. ${ }^{58}$ Die Zeichentheorie bemüht sich zwar, Schrift und Bild auf gemeinsamer Grundlage zu

${ }^{54} \mathrm{Zu}$ der Zuordnung Bild/Kunst/Macht siehe z. B. STEFAN GERMER/MICHAEL ZIMMERMANN (Hrsg.), Bilder der Macht - Macht der Bilder. Zeitgeschichte in Darstellungen des 19 Jahrhunderts. München 1997; FRANKENBERG/NIESEN, Bilderverbot (Fn. 1); ROHL, Visuelle Zeitenwende (Fn. 1), 339.

$55 \mathrm{Vgl}$. zum Beispiel die Ausführungen Jacques Derridas zur Herrschaft der sprachlichen Form in DERRIDA, Grammatologie (Fn. 26), $42 \mathrm{f}$.

${ }^{56}$ Der Bezug von Bild zu Kunst in der Hermeneutik wird vor allem über die Fokussierung auf das Tafelbild deutlich: HANS GEORG GADAMER, Wahrheit und Methode. Grundzïge einer philosophischen Hermeneutik. Tübingen 1990, 157.

57 In der juristischen Argumentationstheorie werden Bilder von vornherein ausgeklammert bzw. nicht thematisiert.

${ }^{58}$ So ist auffällig, dass Luhmann die „Die Kunst der Gesellschaft" vor allem am Betrieb der bildenden Kunst entwickelt. Schon der Literaturbetrieb und die Praxis der musikalischen Aufführung treten in den Hintergrund. Diesen Hinweis verdanke ich Gerhard Plumpe. 
beschreiben, ihr Modell der "Offenen Kommunikation" ist aber das Kunstwerk ${ }^{59}$ und damit folgt sie noch ganz den parallel laufenden Unterscheidungen von Schrift/Bild, geschlossene/offene Kommunikation, Verbindlichkeit/Unverbindlichkeit, Recht/Kunst, Freiheit und Zwang. Auch wird nur der Kunst eine besondere Bedeutung als dem „Königsweg zu einer historischen Anthropologie der Einbildungskraft" zugewiesen ${ }^{60}$ oder es wird die Vorstellung geäußert, Bilder in der juristischen Kommunikation hätten eine "subsemantische Bildwirkung", auf denen die magische "Macht des Bildes" beruhe. ${ }^{61}$ So unterschiedlich die Gedächtniskonzeptionen und die Konstruktion von Referenzstrukturen zwischen den einzelnen wissenschaftlichen Disziplinen sind: die Identifikation von gesellschaftlichen Teilbereichen und Leitmedien prägt die Idee von der Stabilität und Instabilität der Referenzstrukturen, etwa in der Vorstellung, Kunstkommunikation sei eher durch Neuheit, Abweichung, Unverbindlichkeit und Beliebigkeit gekennzeichnet als Rechtskommunikation. Und so werden nicht nur die visuellen Bildträger, sondern auch Sprachbilder und künstlerische Strategien programmatisch aus der juristischen Kommunikation verdrängt oder sie werden ignoriert.

\section{Autonomie und Anästhesie}

In der geschilderten Beobachtungseinschränkung liegt ein Zusammenhang von Autonomie und Anästhesie. Behauptete Autonomie verlangt, dass visuelle Bilder verstummen und Sprachbilder blind werden. Aber dann liegt der Schluss auch nahe, Bilder hätten in der Rechtskommunikation eine subsemantische und magische Wirkung. Die Annahme, das Methodenideal der Rechtswissenschaft sei bilderfeindlich geworden, setzt nicht nur voraus, dass man auch rhetorische Figuren bis hin zur Metapher als Sprachbilder versteht, insoweit man sie zu den basalen Medien des Bildes zählt und aus den Kommunikationsbedingungen des Rechtes ausschließt; es setzt auch voraus, dass man Rechtsbegriffen auch dann noch ihre bildliche Qualität abspricht, wenn sie so natürlich klingen wie Rechtsquelle, so architektonisch wie Rechtsgrundlage oder so musikalisch wie Harmonisierung des Europarechts. Schließlich setzt die Annahme herrschender Bilderfeindlichkeit voraus, dass die Dynamik

59 UMBERTO ECO, Das Offene Kunstwerk (1962). Frankfurt am Main 1993.

${ }^{60}$ Hans Ulrich RecK, Kunst durch Medien, in: WOlFGang MÚller-FunK/Hans Ulrich RECK (Hrsg.), Inszenierte Imagination. Beiträge zu einer historischen Anthropologie der Medien. Wien 1996, 45.

61 Vgl. RÖHL (Fn. 1); Helmut SCHUlzE-FIELITZ, Notizen zur Rolle der Verwaltungsrechtswissenschaft für das Bundesverfassungsgericht, in: DÖV 2003, 421 ff., 444. 
der Formgebung stillgestellt wird und Metapher Metapher und Argument Argument bleibt. Eine Metapher ist eine junge Tatsache und eine Tatsache ist eine alte Metapher, so Nelson Goodmann. Die Stillstellung mit ihrem Gewinn der Stille gelingt also nur zum Preis der oben beschriebenen "Veraltung der Rechtswissenschaft". Nun ist Recht eine Erscheinung, die zeitlich ist und daher der Alterung unterliegt. In diesem Sinne kann man Rechtsbegriffen mal eine stärkere, mal eine schwächere Metaphorizität und damit eine stärkere und eine schwächere Bildlichkeit bescheinigen, wie etwa in der Beobachtung, dass sich Rechtsmetaphern in Katachresen verwandeln können und ihre Bildlichkeit verblassen kann.62 Damit wechselt man aber nur von Problemen der Form zu Problemen der Zeit. Konsequenterweise muss man dann mit rechtssystematischen Grenzen auch Epochengrenzen bestimmen und in diesem Wechsel von Zeitproblemen zurück zu Formproblemen Zeiträume schaffen.

Ein Beispiel hierfür ist die oben erwähnte hegelianische Beschreibung der Entstehung des bürgerlichen Rechtsstaates und des Endes der Kunst. Die Konstruktion dieses Rechtsstaates als nüchterne, anästhetische Erscheinung hängt direkt damit zusammen, dass Hegel die Ästhetik auf ein Problem des Kunstschönen reduziert und hiermit eine Entwicklung benennt, in der Bildproduktion als Synonym zur Kunstproduktion verstanden wird und zugleich ein Großteil visueller Produktionen ignoriert wird, unbeobachtbar bleibt. Das ist eine Entwicklung, in der einerseits die Ikonologie/Ikonographie verstummt sowie andererseits die Gesellschaft bildblind oder besser bildanalphabetisch wird. Soweit visuelle Produktionen in der Moderne noch als Bilder wahrgenommen werden, so zum Preis der Absage an die Referenz, also entweder als "abstrakt-sprachlose" Bilder wie Barnett Newmanns Who's afraid of red, blue and yellow? oder als ikonographisch beliebig und unverbindlich redende Bilder wie Joan Mirós Woman and bird in the night. ${ }^{63}$ Die Ikonophobie des modernen Rechts findet ihre Entsprechung in der Referenzangst des modernen Kunstbetriebes. Vor allem im Betrieb der bildenden Kunst hat sich die Vorstellung ausgebildet, Bilder seien sprachlos. Auch die Kunstgeschichte hat diese Vorstellung wohl durch die Entgegensetzung von formaler Ästhetik und Stilanalyse einerseits und Ikonographie/Ikonologie andererseits weiter befördert. Die Bildpraxis der Vormoderne mit ihren Emblemen, kommentierten Herrscherbildnissen und ihrer Tradition der Ikonographie/Ikonologie unterschied im Hinblick darauf, wie Kommunikation an Kommunikation anknüpft, nicht in dem strikten Sinne, wie es in der Moderne ge-

\footnotetext{
${ }^{62}$ StEINHAUER, Princeps iustitiae advigilans (Fn. 48).

${ }^{63}$ Abbildung und Besprechung z. B. in STOLLEIS, Das Auge des Gesetzes (Fn. 1), 14.
} 
schieht. ${ }^{64}$ Erst die Entwicklung der Vorstellung autonomer Kunst ist eine der Voraussetzungen für das Verstummen des Bildes. ${ }^{65}$ Es ist eine in der Kunstgeschichte schon länger beklagte Tradition, dass die meisten visuellen Produktionen des 19. und 20. Jahrhunderts daher nicht zum Gegenstand der Kunstgeschichte wurden. Die Ausnahmen, wie Aby Warburgs Bildatlas, blieben gleichwohl lange Zeit einmalig und die Aufarbeitung dieses Problems der Kunstgeschichte unter dem Begriff des Iconic Turn und der Begründung einer Bildwissenschaft ist noch nicht die Lösung des Problems, solange diese Bildwissenschaft nicht anderes als modernisierte Kunstwissenschaft ist. Das Problem liegt auch hier weder einfach in der Frage, was Kunst ist, noch in der Frage, was Recht ist, sondern in der Kollision unterschiedlicher gesellschaftlicher Rationalitäten, ihren selbstdisziplinierten Formgebungstraditionen und darin, wie das Recht Kunst und Bilder und wie Kunst und Bilder das Recht beobachten können - ob also für diese Kollisionen Entscheidungsmechanismen zur Verfügung stehen. Die Voraussetzung, die moderne Gesellschaft sei durch die Autonomisierung gesellschaftlicher Teilbereiche gekennzeichnet, erschwert eher diese Beobachtungen, sie ist damit eine Art der Anästhesie. ${ }^{66}$ Der Topos, der Kunstbegriff der Moderne sei durch die Abwendung von Regelprinzipien bestimmt, ist nicht nur Grundlage der Vorstellung künstlerischer Autonomie, sondern hält sich nur solange man ignoriert, dass mit der Verrechtlichung des Bilderstreites erst in der Moderne rechtliche Superregeln für die Kollision von Darstellungsansprüchen und Selbstbestimmungsansprüchen geschaffen wurden (z. B. \$22 KUG, § 823 I BGB i.V.m. § 1004 BGB, Art. 5 Abs. 1 und Abs. 3 GG und Art. 2 Abs. 1 GG), die ältere, nicht justiziable Regulierungsmuster wie die Produktionsregeln an den französischen Akademien abgelöst haben. Diese Einschränkung der Beobachtungsbedingungen ist umso weniger zufrieden stellend, als die Autonomie der Bildproduzenten gleichwohl auch nicht im Schutzbereich einer vorbehaltlos gewährleisteten Kunstfreiheit gesichert ist. Was die Bildwirtschaft heute als Bilder produziert und handelt, wird weitgehend nicht als Kunst geschützt. ${ }^{67}$ Die Behandlung von Bildkonflikten

64 LUHMANN, Die Kunst der Gesellschaft (Fn. 32), $32 \mathrm{ff}$

65 Vgl. NORBERT BOLZ/UlLrich RÜFFer (Hrsg.), Das große stille Bild. München 1996.

$66 \mathrm{Vgl}$. Fabian SteinhaUer, Rezension zu Günther Frankenberg/Peter Niesen (Hrsg.), Bilderverbot. Recht/Ethik und Ästhetik der öffentlichen Darstellung, in: KJ 2004, 373379 .

67 Vgl. die Darstellung der Bildwirtschaft bei MATTHIAS BRUHN, Bildwirtschaft. Verwaltung und Verwertung der Sichtbarkeit. Weimar 2003. In dem juristischen Standardwerk zu Äußerungs- und Formulierungsrechten (,Wenzel ${ }^{\prime \prime}$ ) finden sich erst seit der 3. Aufl., allerdings zunächst nur marginale Ausführungen zur Kunstfreiheit, anders erst: EMANUEL BURKHARDT/WALDEMAR GAMER/JOACHIM RITTER VON STOBL-AlbeG, Das 
ist dabei kaum rechtssystematisch gehegt, wenn man an die widersprüchliche und nicht vorhersehbare rechtliche Behandlung visueller und textlicher Darstellungen im Spannungsfeld von Werbung, Kunstbetrieb, Autobiografien, Regenbogenpresse und Schlüsselromanen denkt.68 Man kann daran erkennen, dass der Bilderstreit als Streit um die Unterscheidung von Codes auch Rückwirkungen auf das Programm des Rechts selbst hat: Die Behandlung von Bildern im Recht gelingt nicht systematisch, solange die Systematik der Abgrenzung des Rechts gegenüber der Kunst unscharf bleibt. In dieser Unschärfe kann man einerseits also eine Überidentifizierung von Bild und Kunst in den Ästhetiken und Gesellschaftstheorien beobachten, andererseits eine Unteridentifizierung von Bild und Kunst in der Rechtswissenschaft und Kunstgeschichte. Beides korreliert nicht.

\section{Monomedialität und Ausdifferenzierung}

In der Praxis der Wissenschaft kann man z. B. autobiografisch solche Widersprüchlichkeiten zwischen den verschiedenen Theoriedesignmöglichkeiten mit Disziplinierungen der Wissenschaften und Selbstdisziplinierung als Wissenschaftler auflösen, entweder also Rechtswissenschaft oder Kunstwissenschaft oder Medientheorie betreiben und den Rest sein lassen. Weder im Hinblick auf die Idee einer Einheit des Wissens ist das aber befriedigend, noch im Hinblick auf die Idee einer Einheit des Rechts. Vor allem die Systemtheorie bemüht sich daher, die Ausdifferenzierung der Teilsysteme unter den Bedingungen ihrer Vergleichbarkeit zu beschreiben. Mit diesem Ausgangspunkt ist der vorliegende Beitrag auch der soziologischen Medientheorie Luhmanns verpflichtet. Dabei gibt es auch in der systemtheoretischen Beschreibung eine Schwäche. Auch Luhmanns soziologische Medientheorie folgt der Deutung, in der die Monomedialität der Kommunikationsbedingungen in gesellschaftlichen Teilbereichen und die gesellschaftliche Ausdifferenzierung als sich gegenseitig bedingende Entwicklung dar-

Recht der Wort- und Bildberichterstattung. 5. Aufl., Köln 2003. Die Entscheidungen des BVerfG zur Werbekampagne von Benetton dringen gleichwohl nicht zur Kunstfreiheit vor, vgl. BVerfGE 102, 347 (Schockwerbung I).

68 „Sinn und Aufgabe des Grundrechts aus Art. 5 Abs. 3 Satz 1 GG ist es vor allem, die auf der Eigengesetzlichkeit der Kunst beruhenden, von ästhetischen Rücksichten bestimmten Prozesse, Verhaltensweisen und Entscheidungen von jeglicher Ingerenz öffentlicher Gewalt freizuhalten", BVerfGE 30, 173 (190). Vergleiche dies mit der Darstellung der Entstehungsbedingungen des Romans inklusive aller Ingerenzen etwa bei MiChael TÖTEBERG, Nachwort, in: KLaUS MANN, Mephisto. Roman einer Karriere. Reinbek bei Hamburg 2003, 392 ff.; siehe zu jüngeren Konflikten auch LADEUR, Anpassung des Medienrechts (Fn. 51), 393 ff. 
gestellt werden. Seine Darstellung des Aufbaus von Referenzstrukturen in der Kommunikation unterteilt zwar nicht nach den unterschiedlichen Trägermedien Sprache und Bild. Der Buchdruck spielt aber vor allem eine Rolle in der Ausdifferenzierung des Rechts. Kunst und Bild werden bei Luhmann beinahe als Synonyme gebraucht und dies verbindet ihn mit der ästhetischen und poetischen Tradition des 20. Jahrhunderts..$^{69}$

Es ist aber gerade medientheoretisch eigenartig, dass in dem $\mathrm{Zu}$ sammenhang von Monomedialität und gesellschaftlicher Ausdifferenzierung gesellschaftliche Unterscheidungen wie die zwischen Recht/ Kunst und Politik auf mediale Unterscheidungen zwischen Schrift und Bild bezogen werden und in ein Bedingungsverhältnis gesetzt werden. Dirk Baecker beschreibt das Problem so:

„Der Fehler setzt dort ein, wo man das Verhältnis von Form zu Medium als notwendiges Bedingungsverhältnis beider Seiten der Differenz nicht respektiert und damit von der kybernetischen Erklärung qua Einschränkung wieder auf die kausale Erklärung im Muster von Ursache und Wirkung zurückfällt und diese nach dem Muster der Medientheorie allenfalls noch dadurch flankiert, daß man die Ursachen unbeobachtbar werden läßt und den Wirkungen selbstverstärkende Rückkopplung konzediert ... Ein Medium schränkt ein, welche Kopplung von Elementen möglich ist, aber es fällt mit der Kopplung nicht in eins. Es bleibt lose Kopplung. ${ }^{.70}$

Hat man sich einmal entschieden, Buchdruck, Sprache, Schrift und Bild nicht nur als Form, sondern auch als Medium zu beschreiben, so muss man konsequenterweise immer gleich mehrere dieser Medien ins Blickfeld nehmen. Mit noch stärkerer Distanzierung von der systemtheoretischen Unterscheidung zwischen Bewusstsein und Kommunikation lässt sich auch etwas anderes einwenden. Im Ensemble aus Autor, Schrift, Sprache, Verleger, Vorleser, Redner (Richter, Anwälte, Verwaltungsbeamte und Rechtswissenschaftler) und Leser und Zuhörer (Rechtswissenschaftler, Angeklagte, Parteien, Mandanten etc.) bleibt sowohl die Stabilisierungs- als auch die Destabilisierungsleistung eines basalen Mediums ungreifbar, weil die Medien nicht bloß in jedem Medium dieses Ensembles (z. B. Buch, Rede, Jurist) Sinn verkörpern, nicht bloß repräsentieren, nicht bloß appellieren, evozieren oder motivieren. Sie erfüllen auch alle diese Funktionen. Der Inhalt eines Mediums ist immer ein anderes Medium (McLuhan). Allein aus den Eigenschaften

69 Siehe auch die Darstellung bei LuHMANN, Die Kunst der Gesellschaft (Fn. 32), 165 ff., 226.

70 Die Kritik richtet Baecker gegen Friedrich Kittlers ",harte Medientheorie“, sie kann aber m. E. allgemein auf die Vorstellung der Monomedialität bezogen werden, BAECKER, Kommunikation im Medium der Information (Fn. 4), 122. 
eines Mediums Stabilisierungs- oder Destabilisierungstendenzen für die Kommunikation herauszulesen, also etwa für die Möglichkeit konsistenten Entscheidens im Recht, erscheint daher in hohem Maße problematisch. Das ist eben der Unterschied zwischen einer Einschränkung der Kopplungsmöglichkeiten und der Kopplung von Kommunikation selbst. Der Kern des Problems liegt im Bezug der Medien zur Kommunikation. In der Kommunikation werden auch monomediale Kommunikationsmittel in die Synästhesien des Gegenübers überführt - Begriffe werden zu Bildern und Bilder zu Gesten, Gesten zu Räumen und Räume zu Foren, Foren zu Begriffen etc. Es gibt sprechende Bilder und sichtbare Worte und sogar eine ",architecture parlante ${ }^{\prime 7}{ }^{71}$ Daher ist es problematisch, einem Kommunikationsmedium zugleich eine monomediale Systematik und so eine bestimmte gedächtnisfördernde/kommunikationsstabilisierende oder gedächtnishindernde/destabilisierende Wirkung zuzuschreiben. In den wechselseitigen Zusammenhängen zwischen basalen Medien, Kommunikationsmedien und Leitmedien zeigt sich, dass der Hinweis auf die strukturierende Kraft einzelner Medien kritisch im Hinblick auf die "Semantik der Medien" zu hinterfragen ist. Der Rückgriff auf Medientheorien innerhalb einer Gesellschaftstheorie ermöglicht nicht, sondern sperrt sich gegenüber den Vorstellungen eines monomedialen gesellschaftlichen Subsystems.

In der Geschichte der Medien fehlt jene systemtheoretische Leichtigkeit der Umprogrammierung unter dem Schutz eines Codes. Die gegenseitige Abschirmung autopoietischer Codes des Rechts, der Kunst und der Politik mit ihrer Absicherung über Kommunikationsmedien setzt Monomedialität voraus. Die von Luhmann geschilderte Geschichte der Medien unter dem Leitbild gesellschaftlicher Ausdifferenzierung ist eine Geschichte von bildtextlicher Vermengung zu monomedialer Aufklärung. Die Rechtsgeschichte ist in diesem Sinne zwar eine Geschichte symbolisch generalisierter Kommunikationsmedien, deren Voraussetzung liegt nach Luhmann aber vor allem in der Geschichte der Schriftsprache als einer intervenierenden Variable und in der Geschichte des Buchdruckes als einem Zweifelsfaktor an der historischen Invarianz der Zeichen. ${ }^{72}$ So überzeugend das ist, dem scharfen Fokus der Systemtheorie sind transmediale Medientechniken unscharf geworden. Deutlich wird dies vor allem in Luhmanns Ausführungen zur Kultur

71 Siehe: CARSTEN-PETER WARNCKE, Sprechende Bilder - Sichtbare Worte. Zum Bildverständnis der frïhen Neuzeit. Wiesbaden 1987; zur sprechenden Architektur siehe auch: EMIL KaUFMANN, Von Ledoux bis LeCorbusier. Ursprung und Entwicklung der autonomen Architektur. Wien 1934; ANDREAS HAUSER, „Architecture parlante" - stumme Baukunst, in: CARLPETER BRAEGGer (Hrsg.), Architektur und Sprache. München 1982, 127.

72 Siehe u. a. NikLAS LUHMANN, Macht. Stuttgart 2003, 6. 
als historischem Begriff, in denen er die Gesellschaftsevolution mit ihrer funktionalen Differenzierung scharf von früheren Gesellschaftsbildungen und ihrer rhetorischen Transmissionsdynamik trennt, ${ }^{73}$ sowie in den minimalen Äußerungen über die Beziehung zwischen Kunst und stratifikatorischer Differenzierung. ${ }^{74}$ Die Schärfe des Rechtsbegriffs wird auch bei Luhmann durch eine Unschärfe des Bildbegriffes gewonnen und andersherum wird die Schärfe des Bildbegriffs nur durch eine Unschärfe des Kunstbegriffes gewonnen. Innerhalb der Geschichte des Paragone und des Bilderstreites lautet die Aufgabe, einen Fokus für die transmedialen Bedingungen an den unscharfen Rändern gesellschaftlicher Ausdifferenzierung zu finden. Es ist eine andere Geschichte und eine Geschichte von anderen Differenzen zu erzählen.

\section{Das Ordnungsmuster decorum}

Rom hat dem Recht nicht nur eine Referenz hinterlassen (nämlich Rom) ${ }^{75}$ sondern auch eine Hyperreferenz, d. h. ein implizites und explizites Regelwerk zur Festlegung, Lösung und Verschiebung von Referenzen, die Rhetorik und ihr Ordnungsmuster decorum. Die europäischen Gesellschaften kannten vor der Phase funktionaler Ausdifferenzierung eine Phase der rhetorischen Einheit, in der die Rhetorik die Transmissionsdynamik sozialer Referenzstrukturen verwaltete, also das, was man zum Beispiel mit Sprachen des Rechts bezeichnen kann. ${ }^{76}$ In dem Sinne, in dem Luhmann von der Ablösung des Interesses an der Rhetorik durch ein Interesse an der Kultur spricht, kann man das verbindende Element dieses Ablösungsprozesses bezeichnen, das in dem Aufbau kommunikativer Referenzstrukturen unter den Bedingungen ihre Verkörperungszwänge liegt. Die Rhetorik verfügte dabei über ein implizites und explizites Ordnungsmuster, das sowohl die Funktion

\footnotetext{
${ }^{73}$ LUHMANN, Kultur als historischer Begriff (Fn. 15), 31.

${ }^{74}$ LUHMANN, Die Kunst der Gesellschaft (Fn. 32), 220.

75 Dazu: PIERRE LeGENDRE, Der Take off des Westens ist ein Gerücht. Gespräch mit Pierre Legendre, in: CORNELIA VISMANN (Hrsg.), Pierre Legendre. Historiker, Psychoanalytiker, Jurist. Berlin 2001, 104; MARIE THERES FÖGEN/CORNELIA VISMANN, Referenz Rom, http://www.mpier.uni-frankfurt.de/Forschung/Mitarbeiter_Forschung/2-fögen-vismann -beschreibung.pdf; MARIE THERES FÖGEN, Referenz Rom in der Evolution der Gesellschaft http://www.mpier.uni-frankfurt.de/Forschung/Mitarbeiter_Forschung/3-fögen-evoluti on.pdf; CORNELIA VISMANN, Unentrinnbares Rom, http://www.mpier.uni-frankfurt.de/ Forschung/Mitarbeiter_Forschung/4-vismann-unentrinnbar\%20_2_.pdf; DIES., Akten (Fn. 23), 10.

${ }^{76}$ LUHMANN, Kultur als historischer Begriff (Fn. 15), 33; zur rhetorischen Einheit siehe auch Fabian SteinHAUer, Was ist kein Medium?, in: RAOUl MÖRCHEN (Hrsg.), Visuelle Komposition. Essen 2003, 67.
} 
stratifikatorischer Steuerung, als auch die Funktion der Eröffnung von Gestaltungs- und Formulierungsmöglichkeiten ${ }^{77}$ bis hin zur Regulierung der Affektkommunikation übernahm und sich in der Ambivalenz zwischen Formulierung und Regulierung bewegte. Luhmann merkt an, dass die Beziehungen "der Kunst zur stratifikatorischen Differenzierung" sicher komplexer sind, "als man es im Rückblick vermuten wür$\mathrm{de}^{\prime \prime}{ }^{78}$ Die Komplexität dieser Beziehung entfaltet sich aber gerade jenseits kunsthistorischer Einhegung in der Geschichte der Rhetorik - hier wurde sie auch immer vermutet.

Das Ordnungsmuster decorum kann als Medientechnik verstanden werden, das sich auf die laufende Umcodierung von Sinn in unterschiedlichen Medien einstellen konnte, und das gilt sowohl für Kommunikationsmedien im Luhmann'schen Sinne wie z. B. Recht oder Macht als auch für die basalen Medien Schrift-Sprache-Bild. Von der Phase der rhetorischen Einheit kann gesprochen werden, solange das Ordnungsmuster decorum seine transmediale Wirksamkeit in rhetorischen Handlungsanleitungen, Fürstenspiegeln, Architektur- und Maltraktaten und in Regelbüchern der Poetik und der Bildproduktion an den Akademien entfalten konnte und damit Handlungsbereiche zwischen "Politik", „Recht" und "Kunst" zu einer Medienpraxis der Formulierungsmöglichkeiten verbunden hat. Nicht nur in den explizit verfassten Regeln der rhetorischen Handlungsanleitungen (vor allem bei Quintilian), ${ }^{79}$ sondern auch in den früheren rhetorischen Mustertexten wie der Verteidigungsrede der Helena von Gorgias und in der Architekturtheorie von Vitruv (um $14 \mathrm{v}$. Chr.) lassen sich decorum-Strukturen in den schriftlichen und in den visuellen Gestaltungsformen der griechischen und römischen Zeit nachweisen, so dass nur schwer zu sagen ist, was eher formuliert wurde, das implizite oder das explizite decorum. ${ }^{80}$ Diese Schwierigkeit hängt unauflöslich mit der Ambivalenz des decorum als einem Ordnungsreflex zwischen gesellschaftlicher

77 Ich werde im Folgenden zur sprachlichen Vereinfachung den Begriff der Formulierungen für alle Formgebungen verwenden, also nicht bloß für Gerichtsreden, politische Reden oder Rechtsätze, sondern auch für Operationen oder Beobachtungen, die als Form beobachtet werden können wie z. B. die Krönung Ludwigs des IX., eine Photographie von Caroline v. Monaco oder ein Demonstrationszug wie die Love- oder die Fuckparade.

${ }^{78}$ LuHMANN, Die Kunst der Gesellschaft (Fn. 32), $220 \mathrm{f}$.

${ }^{79}$ MARCUS FabIUS QuiNTILIANUS, Ausbildung des Redners. Zwölf Bücher (zwischen 35-100 n. Chr.). Darmstadt 1972.

${ }^{80}$ Ursula Mildner merkt dazu an: „Für Malerei und Architektur der Antike ist eine der Rhetorik vergleichbare Systematik des decorum nicht überliefert; die verstreuten Angaben zur bildenden Kunst lassen aber seine deutliche Anwendung erkennen." URSUla MILDNER, decorum, in: GERT UeDING (Hrsg.) Historisches Wörterbuch der Rhetorik. Bd. 2. Tübingen 1994, 434 
Regulierung und medialer Formulierungspraxis zusammen. Das dem decorum bescheinigt wird, flexibel aber verschwommen, ungenau und unverbindlich zu sein, ${ }^{81}$ ist wohl auch eher diesem Reflexionsmodus als dem Umstand der historischen Langlebigkeit zu verdanken. Man verkennt den für den Aufbau von Referenzstrukturen so wichtigen Reflexionsmodus, wenn man das decorum aufgrund der Annahme, es sei unverbindlich und nicht justiziabel, ignoriert oder als „Vorraum der Moral" (Asmuth) marginalisiert. Das decorum bot mit dieser Reflexionsleistung die Möglichkeit, über die Kollision unterschiedlicher Formgebungsmöglichkeiten zu streiten und sie zu beobachten. Mit seinem Fokus lieferte das decorum damit eine Technik der Aufmerksamkeit, wie sie Theorien eben zur Reflexion der Praxis bieten sollen.

Die römische Hyperreferenz erhält nach der antiken Ausgestaltung in den rhetorischen Lehrbüchern vor allem im Humanismus, der ästhetischen Theorie der Renaissance und in der französischen Klassik eine explizite Ausprägung, die über die Architektur, das Zeremoniell und die Ikonologie die Kommunikationsbedingungen des Rechts in der proto- und frühkonstitutionellen Phase modulierte. ${ }^{82}$ Damit sind über Zeiträume hinweg drei zentrale Elemente des decorum vorzustellen:

\section{Differenzierung als Relationierung}

Das decorum dient innerhalb der Formgebungsdynamik der Kommunikation der Relationierung und Differenzierung der Medien. Als medientechnischer Wertbegriff verlangt das decorum Relativierung (wertvoll im Hinblick worauf?), die zugleich als medienimmanente Relationierung umgesetzt wird. Wenn man über eine historisch weit reichende Phase wie die seit der Niederschrift früher rhetorischer Texte bis hin zur Regierungszeit Ludwig des XIV. Im 18. Jahrhundert und ihrer Nachwirkung auf die französische Klassik und die Staatenbildung in Europa nur eine Funktion des decorum benennen will, dann war es die, über die Angemessenheit von Formulierungen zu entscheiden und dafür eine Technik bereitzustellen, mit der in den Medien Relationen markiert und Differenzen freigesetzt werden konnten. Man kann zwischen einer ethischen, einer ästhetischen, einer rhetorischen und einer

81 Vgl. z. B. Ramus, Rhetoricae Distinctiones in Quintilianum (1549), Illinois 1986, 158; dazu auch IAN RUTHERFORD, decorum, in: UEDING, Rhetorik (Fn. 80), 423, 432.

82 Dazu: HeINER MÜhlmanN, Leon Battista Alberti. Ästhetische Theorie der Renaissance. Bonn 1981; DERS. Über den humanistischen Sinn einiger Kerngedanken der Kunsttheorie seit Alberti, in: Zeitschrift für Kunstgeschichte 33 (1970), 127-142; MILOS VEC, Zeremonialwissenschaft im Fürstenstaat. Studien zur juristischen und politischen Theorie absolutistischer Herrschaftsrepräsentation. Frankfurt am Main 1998. 
juristischen Dimension des decorum unterscheiden, die Funktion des decorum betrifft aber jede Formgebung in der Kommunikation, unabhängig davon, ob es sich um "Rechtskommunikation", "politische" Kommunikation, "künstlerische" oder "moralische" Kommunikation handelte. Unter der Beobachtungsperspektive einer Geschichte des decorum oder einer Geschichte der "öffentlichen“ Darstellungspraxis erscheinen die Unterscheidungen nach gesellschaftlichen Teilbereichen wie Recht/Politik/Kunst als Differenzierungen, die in der Relationierung gleich wieder aufgehoben werden.

Das decorum ist eine rhetorische Kategorie, die das Schicksal aller rhetorischen Konstanten teilt. Es ist die "mysteriöse Weichheit fester Formeln", die das Latein latent als Garant bewahrt und der jede Epoche, jede Schule, jede Richtung ihren eigenen Inhalt gibt (Munteano). Die Funktion der Regulierung von Angemessenheit und Relationierung von sprachlichen, schriftlichen oder visuellen Formulierungen (Gestaltungen) hält sich als Konstante durch die Geschichte des decorum aber durch. So kann man in den früheren schriftlich überlieferten Zeugnissen der griechischen Literatur beobachten, wie die Relationen zwischen Stil und Gegenstand, zwischen Sprache und Charakter, zwischen Rede und Publikum, zwischen Rede und Zeitpunkt und zwischen Ornament und Inhalt markiert und damit bestimmte Differenzen freigesetzt und damit für Rede-, Schrift-, und Bildtechnik verfügbar werden ${ }^{83}$ Der rhetorische Kern des decorum liegt darin, dass mit Relationierung und Differenzierung jedes Thema als die Modulation eines anderen Themas verstanden werden kann. In den Figurenbildungen der Metaphoneme, Metamorpheme, Metataxeme und Metasemene läuft dabei eigentliches und uneigentliches/bildliches Sprechen zusammen. In diesem Sinne ist das decorum mehr Hyperreferenz als Referenz, weil es nur ein Ordnungsmuster für Relationierungen, d. h. Referenzbildungen liefert, ohne selbst Referenz zu sein.

Das decorum ist Medientechnik, weil es im oben geschilderten Sinne der Medientheorie eine Figur/Grundbeziehung schafft. Dabei handelt es sich zugleich um eine einheitsbildende Operation über das Verhältnis eines Teils zum Ganzen, mit dem etwa das Verhältnis eines Satzes zum Zweck der Rede oder einer Entscheidung zum Ziel der Gerechtigkeit bestimmt wurde. Das decorum verfügt damit über einen Maßstab

${ }^{83}$ Zur Relationierungen zwischen Sprache und Gegenstand siehe den Mustertext von ARISTOPHANES, Frösche. Frankfurt am Main 1971, 1058 f.; ISOKRATES, Panegyrikos, 8 ff.; PLATON, Phaidros, 267a, zwischen Sprache und Charakter siehe PLATON, Ion, 540 b ff.; DeRS., Politeia 3 400d; ArISTOTELES, Poetik, 1454a 30; zwischen Rede und Publikum siehe Platon, Phaidros, 271d ff.; zwischen Rede und Zeitpunkt THUKYDIDES, Geschichte des Peleponnesischen Krieges. Bd. 1. Leipzig 1961, 35; siehe zur frühen Entwicklung des decorum auch RUTHERFORD, decorum (Fn. 81), 423. 
für den Umgang der Korrelation zwischen Einheit und Differenz. Das decorum markiert Relationen, setzt damit Differenzen frei und macht diese verfügbar. Es ist systembildend durch einheitsstiftende Wirkung unter den Bedingungen komplementärer Differenz. Im Bereich des "Kunstsystems" ist dies in den Malerei- und Architekturtheorien abzulesen, in denen das decorum Erscheinungsweise des Individualschönen ist und einen Bezug zwischen Binnenstruktur des Werkes und seiner Außenwirkung in der Umgebung schafft. Im Bereich jener Kommunikationen, die aus heutiger Sicht am ehesten als Rechtskommunikation beschrieben werden könnten, bezieht sich diese Funktion auf den zum Einheitssymbol des Gerechten komplementären Maßstab des Billigen (iustum/aequum) ${ }^{84}$ Bei aller Systembildung behält das antike und das humanistische decorum ,einen gesellschaftlichen Sinn, eine gesellschaftliche Sinnlichkeit, die auf das Konkrete gerichtet ist" ${ }^{\prime 85}$ Nur mit einer gewissen Distanzierung vom Systembegriff Luhmanns kann man hier daher von systembildender Leistung sprechen, weil das decorum in gesamtgesellschaftlicher Perspektive immer in der Spannung der Totalität der Gesellschaft und dem Fragment einzelner gesellschaftlicher, nicht systematisch geschlossener Ausprägungen stand.

\section{Differenzierung als Polarisierung}

Mit dem Bereich gesellschaftlicher Sinnlichkeit ist das zweite Element des decorum hervorzuheben. In dem Maße, in dem das decorum als Ordnungsmuster zur stratifikatorischen Steuerung der Gesellschaft beitrug, hatte es zugleich eine "politische" Funktion. Die Operationen der Relationierung und Differenzierung, die das decorum vollzieht, sind hier zugleich eine Operation der Polarisierung. Mit Polarisierung ist im Gegensatz zur Differenzierung die Verschärfung einer Grenzziehung gemeint, die ihre Bedeutung darin hat, dass sie sich nicht nur im Medium der Information (Baecker), sondern auch im Medium der Emotion/des Affekts vollzieht, in der eine Hierarchie der Stilqualitäten mit einer gesellschaftlichen Hierarchie verknüpft wird. ${ }^{86}$ Die Systemtheorie Luhmanns blendet diese Sinnlichkeit der Kommunikation aus, weil Kommunikation ein operativ geschlossenes System ist. Aber es ist

\footnotetext{
84 Siehe dazu MühlmanN, Alberti (Fn. 82), 21 ff., 37 ff.

85 MÜHLMANN, Über den humanistischen Sinn (Fn. 82), 129.

86 Unter Hinweis auf den Rechtshistoriker Pierre Legendre und seinem Interesse an den Bildern in der Sprache des Rechts spricht Peter Goodrich von dem „emotional body of law". Siehe dazu: PETER GOODRICH, Languages of Law. From Logics of Memory to Nomadic Masks. London 1990, 260.
} 
fraglich, warum Buchdruck ein Medium der Kommunikation sein soll, nicht aber der Körper der Kommunikationsteilnehmer.

Die Bedingung des decorum als eines polaren Ordnungsmusters wird in dem Wandel der aristotelischen zur römischen Rhetorik, vor allem an der Rhetorik an Herennius, expliziert. ${ }^{87}$ Deutlich wird dies an der Rolle des pathos in der Rhetorik. In der Rhetorik des Aristoteles ist pathos noch der unregulierte Affekt. In der römischen Tradition wird pathos in die Dreistillehre des decorum einbezogen und es bezeichnet hier den hohen Stil, das Erhabene, gegenüber dem ethos des mittleren Stils. Die Dreistillehre als Element des decorum ist in der antiken Rhetorik erst in der Rhetorik an Herennius greifbar. Früher, wie etwa bei Aristoteles, fehlt noch die Vorstellung eines mittleren Stils, ebenso die Korrelationen zwischen mittlerem Stil und ethos sowie hohem Stil und pathos. Dieser Wandel mag auf einem römisch verstandenen Eigenwert der Verschärfung einer Grenzziehung und ihrer affektiven, performativen und sinnlichen Konstituierung beruhen. ${ }^{88}$ Aber auch nach dieser römischen Einbindung des Affektes in die Dreistillehre bleibt ein Rest des unregulierten Affektes zurück, weil sich das Erhabene am Rande des Regulierbaren und Darstellbaren selbst bewegt.

Das decorum verwendet also die polarisierte Skala der Dreistillehre, nach der Formulierungen folgendermaßen unterschieden werden können:

$$
\text { erhaben - was dazwischen liegt - niedrig }
$$

Mit dem decorum wurde eine komplexe Topologie der Formulierungsmöglichkeit in unterschiedlichen Räumen, an unterschiedlichen Orten und $\mathrm{zu}$ unterschiedlichen Zeiten entwickelt. Formulierungen sind in allen Stilhöhen möglich, aber nur bedingt durch die richtige Relationierung im mehrfach verschachtelten Raum einer totalen Gesellschaftsperspektive.

Das decorum erfüllt auch hier die Funktion einer Relationierungstechnik. Das Besondere bei der Funktion der Polarisierung liegt aber darin, dass sie als Erstes in Abhängigkeit zur ständisch organisierten Gesellschaft stand, in der der höchste Wert zugleich eine gesellschaftliche Grenze markiert. Differenzierung wird dabei zur Polarisierung, wenn die Relationierungen in der Kommunikation nicht nur gesellschaftsintern auf gesellschaftliche Hierarchien bezogen, sondern zugleich über den höchsten Grenzwert auch externalisiert werden können auf das Außen der Umwelt. Wenn man Europa für die Welt hält, dann

\footnotetext{
87 ANONYM, Rhetorica ad Herennium. Düsseldorf 1998, IV 8, 11.

88 Dazu: CHRISTINE PRIEs, Das Erhabene, in: GERT UEDING (Hrsg.), Historisches Wörterbuch der Rhetorik. Bd. 3. Tübingen 1996, 1357.
} 
kann man sagen, dass das rhetorische decorum immer ubiquitär, nie aber global war, weil der höchste Wert des decorum immer auf die Grenze der eigenen Stadt oder des eigenen (höfischen) Wirkungskreises bezogen war. Es galt überall, aber überall nur im eigenen politischen Raum. ${ }^{89}$ In diesem Sinne verschachtelte das decorum den politischen Raum.

Der Idealtypus hierfür wird in der ästhetischen Theorie der Renaissance und im Fürstenstaat der frühen Neuzeit gefunden.

"Ausgehend von der gesamtgesellschaftlichen Vorbildlichkeit der höfischen Umgangsformen verbindet sich das Ordnungsmuster Ceremoniel mit dem Instrumentarium des absolutistischen Interventionsstaates. Sämtliche ständischen Differenzierungen, die ihren Niederschlag in den Policey-Ordnungen finden, werden auf diese Weise prinzipiell zeremonialwissenschaftlich legitimiert. Es entsteht auf der Grundlage kosmologischer Ordnungsvorstellungen, naturrechtlich gedachter Rangverhältnisse und eines statischen Geschichtsbildes die utopische Vorstellung einer hinsichtlich ihrer Selbstdarstellung lückenlos normierten Gesellschaft, die die Ehrkonflikte einhegt und das decorum zu staatlichen Zwecken instrumentalisiert. Das Ceremoniel erscheint als ständeübergreifender, gesellschaftsstabilisierender Ordnungsbegriff." ${ }^{\prime \prime 0}$

Der erhabene Körper ist im decorum mit der Simulation der majestas (Milos Vec) der Körper des Königs. Das Erhabene der Grenzmarkierung findet sich aber auch analog in der Architekturtheorie Albertis, in der der erste Sakralbau nicht der Tempel als Ort des göttlichen Rechts ist, sondern das Stadttor als Grenze des Rechtsraums. ${ }^{91}$ Die Polarisierung des decorum zieht ihre strukturierende Kraft aus einer Hierarchie, die auf eine Unterscheidung hinausläuft und die am Ernstfall geeicht ist. Mit den Worten Carl Schmitts kann man sagen, dass es die Unterscheidung zwischen Freund und Feind ist oder die Unterscheidung zwischen Regel und Ausnahmezustand. Diese Aussage muss aber dahingehend relativiert werden, dass sie selbst wieder einem Pathos der Formulierung folgt und damit auch anders formuliert werden könnte.

\footnotetext{
${ }^{89} \mathrm{Vgl}$. am Beispiel der Darstellung Apolls: FRANK BÜTTNER, Die Sonne Frankens. Ikonographie im Treppenhaus der Würzburger Residenz, in: Münchner Jahrbuch der bildenden Kunst 30 (1979), 159, 173.

90 VEC, Zeremonialwissenschaft (Fn. 82), 404.

${ }^{91}$ Siehe dazu das siebte, achte und neunte Buch von Albertis De re aedificatoria, in denen die Rangordnung von Sakralbauten, öffentlichen Bauten und Privatbauten entfaltet wird, LEON BATTISTA ALBERTI, De re aedificatoria. Milano 1966, 531; dazu unter vergleichenden Gesichtspunkten WOLFGANG NEUBER, Sichtbare Unterwerfung. Zu den herrschaftsstrategischen Raumvorstellungen in frühneuzeitlichen Idealstadtentwürfen und Utopien, in: CORNELIA JOCHNER, Politische Räume. Stadt und Land in der Frïhneuzeit. Berlin 2003, 1; zur Bedeutung des Stadttors siehe auch CORNELIA VISMANN, Akten (Fn. 23), 31.
} 
Die Entwicklung des juristischen Souveränitätsbegriffs aus der rhetorischen Tradition der majestas kennt viele andere Unterscheidungen (z. B. Recht/Unrecht oder gebundene/ungebundene Souveränität), deren analoger Charakter zu der Schmitt'schen Unterscheidung in Abhängigkeit vom decorum hervor- oder zurücktritt, je nachdem, in welcher Stilhöhe argumentiert wird.

\section{Transmedialität}

Auch wenn man die Ausprägungen des decorum verschiedenen Disziplinen und gesellschaftlichen Teilbereichen zuordnen kann, dem Eigensinn des decorum als Ordnungsmuster möglicher Referenzstrukturen und seiner kohäsiven Eigenkraft zur Verschärfung von Grenzziehungen wird man dadurch nicht gerecht. Für die Unterscheidung nach gesellschaftlichen Teilbereichen wie Recht und Politik und damit verbundene Kommunikationsmedien wie Macht oder Recht hatte ich das schon angemerkt, zugleich betrifft diese Kohäsionstechnik aber auch die basalen Medien Schrift, Sprache und Bild. Aus dem decorum folgt die Topologie einer Medienpraxis, die nicht auf eines der basalen Medien (Schrift-Bild-Sprache) beschränkt war, sondern auf eine laufende Umcodierung zwischen Text und Bild in unterschiedlichen Medien ausgerichtet war. Auch wenn sich schon in der Begriffsgeschichte des griechischen prépon ein ausschließlich optischer Sinn zu einem synästhetischen Sinn wandelt, der schließlich durch die lateinische Übersetzung decorum ethisch wird, so erhält sich die Vieldeutigkeit zwischen Visualität und Sprache durch das Ordnungsmuster decorum..$^{92}$ Schon in den schriftlich fixierten Handlungsanleitungen der Rhetorik laufen alle basalen Medien über die Grenzen von Bild, Schrift und Rede zusammen: Der Redner greift eben nicht nur auf die schriftlich fixierten Handlungsanleitungen zurück, er ist auch gestisch bewegter Körper im Raum der griechischen und römischen Städte. Er ist Figur auf dem Grund des Forums mit seinen Säulenordnungen. Die Rhetorik folgt mit ihren Bild- und Dichtkünsten dem dictum Horatii: ut pictura poesis, ut

\footnotetext{
92 Bei Homer ist der Gedanke noch ausschließlich als Verb zu fassen (prépo) und bezeichnet (nach einer Übersetzung von Bernhard Asmuth) die ,in die Augen fallende äußere Erscheinung". Bei Aischylos und Pindar wird es zu einem Wert- und Normbegriff, über das, was dem Träger der Erscheinung "ansteht". Als rhetorischer Fachbegriff ist es erst bei Aristoteles (z. B. Poetik, 15) erkennbar. Im lateinischen decorum setzt sich dazu das Zusammenspiel von optischem Sinn und rhetorischer Schrift, Rede- und Bildtechnik fort, vor allem in der Konkurrenz zwischen decorum und aptum. Siehe dazu BERNHARD ASMUTH, Angemessenheit, in: UEDING, Rhetorik (Fn. 80) 579, $580 \mathrm{f}$.
} 
poesis pictura.93 Eine Rangordnung zwischen poesis und pictura wird dabei aber nicht aufgestellt. Die Bedingung der beiden erstgenannten Qualitäten des decorum ist eben die Transmedialität des decorum. Das kann man sich an der einleitend erwähnten Metapher des Stufenbaus verdeutlichen. Bevor diese Metapher mechanisch auf die Einheit des Rechts bezogen wurde, war sie Metapher des Tugendweges. Die Reichsidee der Schönborns ist in keiner Verfassungsurkunde formuliert, wohl aber in der Gestaltung des Treppenhauses der Würzburger Residenz. ${ }^{94}$ Daraus auf ein Primat des Visuellen im Fürstenstaat zu schließen, wäre wohl typischer Fehlschluss einer Theorie der Bilderflut, die die Vormoderne als Präfiguration der Nachmoderne heranziehen möchte. Dem Ordnungsmuster decorum kommt nur die Aufgabe zu, zwischen ikonographischen und semantologischen Rangordnungen $z u$ vermitteln. Aber erst in dieser synästhetischen Vermittlungsleistung entsteht das Treppenhaus als Ort der Repräsentation und der Kommunikationsraum mit seinen Anschlussmöglichkeiten. Denn ohne einen Rückgriff auf die Literatur von Hofikonologen wie Conrad von Albrecht, der auch das Concept für die Gestaltung der Reichshofkanzlei in Wien formulierte, lassen sich die für die Bestimmung des decorum wichtigen Angaben nicht finden. ${ }^{95}$ Auch das decorum im Treppenhaus der Würzburger Residenz erschließt sich als Ausprägung der Schönborn'schen Reichsidee nur, wenn man das Bildprogramm des Autors Johann Seyfried und die staats- und kirchenrechtliche Lehren in Würzburg liest.

Eine rechtshistorische Beschreibung des decorum lässt sich daher vielleicht auf die Literatur der Fürstenspiegel von Autoren beschränken, die im engeren Sinne der Rechtsgeschichte zuzuordnen sind. Der Geschichte des decorum wird man damit aber nicht gerecht, wenn man zum Beispiel aus dem Abbruch einer bestimmten literarischen Gattung der Fürstenspiegel auf des Ende des decorum schließt. ${ }^{96}$ Schließlich

93 HORAZ, Ars poetica, 361; zur Wechselwirkung von poesis und pictura siehe auch STEFAN GREIF, Die Malerei kann ein sehr beredtes Schweigen haben. Beschreibungskunst und Bildästhetik der Dichter. München 1998; BRASSAT, Das Historienbild (Fn. 47), XV ff.; WARNCKE Sprechende Bilder (Fn. 71); ULRICH HEINEN, Rubens zwischen Predigt und Kunst. Der Hofaltar fiir die Walburgenkirche in Antwerpen. Weimar 1996.

94 Ausführlich dazu: PETER STEPHAN, Im Glanz der Majestät des Reiches. Tiepolo und die Würzburger Residenz. Weißenhorn 2002.

95 STEPHAN, Im Glanz der Majestät (Fn. 94), 176

${ }^{96} \mathrm{Zu}$ diesem Schluss siehe VEC, Zeremonialwissenschaft (Fn. 82), 406, der davon ausgeht, dass die Trias von justum, honestum und decorum sich auf die Dichotomie von Recht und Moral reduziert hat. Wie Habermas im Strukturwandel der Öffentlichkeit erklärt Vec diesen Wandel auch dadurch, dass das bürgerliche Kaufmannsideal das bisherige Leitbild des Aulicus politicus ersetzt. Wie sehr sich aber das bürgerliche Kaufmannsideal diesem Aulicus Politicus assimiliert, ist $\mathrm{zu}$ sehen in der anglo- 
entwickelt sich im ausgehenden 17. und im 18. Jahrhundert an den französischen Akademien die französische Klassik, deren rhetorische Unterscheidungen der Gattungen mit Mitteln des decorum nicht nur den Repräsentationszwecken des französischen Hofes Rechnung tragen. Das Zeitalter der beginnenden Ausdifferenzierung des öffentlichen Rechts ist nicht zufällig das Zeitalter der französischen Klassik und ihrer idealtypischen Ausprägung des decorum. Die Abstraktion der personalisierten Herrschaftsverbände zum verdinglichten Staat ist eine Assimilations- und Dissimilationsphase politischer Symbole und rechtlicher Sprache. Die Metamorphose der Herrschaftsverbände zum Staat schafft sich das idealtypische decorum als Medientechnik der Umcodierung. Ist der Staat erst mal gelungen, ist das decorum vielleicht verzichtbar. Ob er aber gelungen ist, ist offen.

\section{Morphologie des Beendens}

Man kann sicherlich verlangen, dass die getroffenen ahistorischen Aussagen zum decorum historisch relativiert und verortet werden. Ich muss hier aber zum Ende kommen. Und doch ist es die stärkste Form der historischen Verortung, zum Ende zu kommen. Ein Ende in der Geschichte kann nie so verstanden werden, dass danach die Dinge verschwinden. Ganz im Gegenteil. Ein historiografisches Ende löst die Dinge nicht auf, es gibt ihnen ihre Form. ${ }^{97}$ Unter Umständen macht es auch Medien zur Form. Die Kunst kennt ihr Ende, der Staat kennt sein Ende und es stellt sich die Frage, ob auch das decorum sein Ende kennt. Seine Auflösung wurde schon oft beschrieben und auch die Trauer der Vollendung findet sich seit Adam Müller, dem Schöpfer des Begriffes Rechtsstaat und einer "Ästhetik der Politik" immer wieder. 98 Aus der Perspektive des Medienrechtes fallen hier Habermas' Ausführungen zum Strukturwandel der Öffentlichkeit ein, in denen vom "En-

amerikanischen Bildnistradition, vor allem bei Benjamin West und John Singleton Copley. Vgl. JOHN SINGLETON COPLEY, Watson and the shark (1778) als Beispiel der Überblendung eines Kaufmannsschicksals in die erhabene Historia der Michaelsikonographie.

${ }^{97}$ Ganz im Sinne der Benn'schen Poetik: „Wenn es fertig ist, soll es vollendet sein - aber was dann?", in: GOTTFRIED BENN, Altern als Problem für Künstler, in: Gesammelte Werke $B d$. 1. Stuttgart 1997, 554; dazu: BAZON BROCK, Altern als Problem für Künstler, in: Macht des Alters. Köln 1998, 19, 21.

98 BENEDIKT KOEHLER, Ästhetik der Politik. Adam Mïller und die politische Romantik. Stuttgart 1980. 
de der repräsentativen Öffentlichkeit" ${ }^{\prime \prime}$ gesprochen wird. ${ }^{99}$ Folgt aus dem Ende der repräsentativen Öffentlichkeit auch ein Ende des decorum? Eine ähnliche Beschreibung des Endes der Repräsentation lieferte 1923 Carl Schmitt mit seinem Text über "römischen Katholizismus und politische Form ". ${ }^{100}$ Schmitts Text ist aber eine rechtspolitische Stellungnahme aus einer Zeit, in der die repräsentative Öffentlichkeit schon längst von der bürgerlichen Öffentlichkeit abgelöst sein sollte. Gleichwohl lebt sein Text von der Symbiose zwischen rhetorischem pathos und politisch-juridischer Repräsentation. Treffend beginnt er seine Ausführungen mit Hinweisen auf den "antirömischen Affekt". Die Gegenfigur des Unternehmers ist im erhabenen Raum der Repräsentation eine unangemessen niedrige Figur wie es der Kaufmann im decorum der comedia dell'arte ist. Entweder man qualifiziert Carl Schmitt als unzeitgemäßen Zeitgenossen, als "verspätete Figur" und seinen Text als "unzeitgemäße Betrachtung", oder aber das decorum hat bis zu Schmitts Zeiten weiter durchgehalten. Aber was ist mit der Rechtsgeschichte des decorum? Wieder lässt sich der Text Schmitts als "unjuristisch" nicht der Rechtsgeschichte zuordnen, aber damit setzt man wieder jene parallele Differenzierung von Text/Bild und Recht/Macht voraus, die man mit dem Fokus auf die Geschichte des decorum bezweifeln wollte. Als Beleg für eine Ausdifferenzierung kann die vieldeutige und selbst tropische Figur Carl Schmitt zumindest nicht dienen. Aber warum auch immer wieder Carl Schmitt? Rechtsgeschichte kann sich schließlich von der Figurenbildung personifizierter Geschichtsdarstellung lösen. Kann sie das?

Jenseits von Carl Schmitt fällt auf, dass im Bezug zwischen Kommunikationsfreiheiten (Art. 5 I GG, Art. 8 I GG) und dem Rechtsstaatsprinzip (Art. $20 \mathrm{GG}$ ) die Rechtssprechung des Bundesverfassungsgerichts Kommunikation mit dem Filter eines politisch-repräsentativen Ordnungsmusters beobachtet. Darauf basiert die Unterscheidung zwischen öffentlich-privat, in ihr tradiert sich zugleich die Rangordnung des decorum. Die Reduktion der Komplexität von Darstellungskonflikten gelingt dem Bundesverfassungsgericht nicht in der Regel, sondern nur in Abwägungen des Einzelfalls. Wenn das Gericht dann entscheidet, ob ein Bildverbot unangemessen ist, dann entscheidet es auch immer wieder mit darüber, ob die streitbefangene Darstellung angemessen ist. Im Sinne des decorum tradiert sich dabei ein Sinn für Angemessenheit (Günther), von dem schwer gesagt werden kann, ob er rational oder irrational ist. Zumindest fügen sich die Aussagen des

\footnotetext{
99 JÜRGEN HABERMAS, Strukturwandel der Öffentlichkeit. Untersuchung zu einer Kategorie der bürgerlichen Gesellschaft (1962). Darmstadt 1979, $17 \mathrm{ff}$.

100 CARL SCHMITT, Römischer Katholizismus und politische Form. Stuttgart 2002.
} 
BVerfG zur politischen Funktion der Kommunikationsfreiheit und zur Themenfähigkeit von $z$. B. Sexualität in die Tradition des decorum, wenn etwa "Love und Fuck" im Schutzbereich von Art. 8 I GG nicht für themenfähig gehalten wird, weil die "niedrigen" Inhalte sexueller Reproduktion/Intimität nicht in den erhabenen Bereich politischer Meinungsbildung und ebenso wenig zum Prinzip repräsentativer Demokratie passen..$^{101}$ Als Spaßparade sind sie aus der Sicht des Bundesverfassungsgerichts dem Ernstfall der Repräsentation unangemessen. Über die bloße Übereinstimmung von Begriffen hinaus schreibt sich die Referenzstruktur des Rechts mit Mitteln des decorum weiter.

Auch jenseits des Zentrums (die barocke Idealstadt Karlsruhe und ihre Rechtssprache) ist in der Peripherie der rechtswissenschaftlichen Literatur ein Bilderstreit zu beobachten. Er knüpft an die Disziplinierung der Rechtsmethode an und ist Ausdruck der aktuellen Bilderfeindlichkeit, etwa im pejorativen Gebrauch des Wortes Metapher. ${ }^{102}$ Das Metaphorische eines Textes wird aber erhöht, wenn er Metaphern ausschliest. Eine von allen Sprachbildern gereinigte Sprache - Wissenschaft ohne Poetik - wird zum Bild schlechthin, wie im logischen Positivismus von Ludwig Wittgenstein und seiner Idee des logischen Bildes. ${ }^{103}$ Die rechtswissenschaftsintern prägnante Unterscheidbarkeit zwischen Argument und Figur und zwischen Text und Bild wird daher nur durch erhöhte Verwechselbarkeit aus der Sicht anderer Disziplinen erreicht. Was dem System Argument ist, ist der Umwelt Figur. Das gilt nicht nur im Verhältnis zur Politik ("Symbolische Gesetzgebung ") oder $\mathrm{zu}$ anderen gesellschaftlichen Ordnungssystemen. Auch innerhalb der Rechtswissenschaft bilden sich Milieus aus, deren Code von anderen Milieus als "bloße Metaphorik" gekennzeichnet und damit ignoriert wird. Der Streit um Metaphern ist aber der Streit um angemessene Bilder. ${ }^{104}$ Wenn das Verstummen der visuellen Bilder und das Erblinden der Sprachbilder eine Bedingung des modernen Rechts ist, dann war es zugleich eine Bedingung des modernen Rechts. Probleme der Form bleiben mit Problemen der Zeit verstrickt, Zeit verstrickt Gerech-

\footnotetext{
101 BVerfG NIW 2001, $2459 \mathrm{ff}$

102 Vgl. als Beispiel MARC AMSTUTZ, Zwischenwelten. Zur Emergenz einer interlegalen Rechtsmethodik im europäischen Privatrecht, in: JOERGES/TEUBNER, Rechtsverfassungsrecht (Fn. 6), 223; andererseits der Vorwurf der Metaphorik gegenüber der Systemtheorie bei ATHANASIOS GROMITSARIS, Rezension von Niklas Luhmanns „Die Politik der Gesellschaft", in: Der Staat 2001, 631 ff., 636 f.; dazu auch: FABIAN STEINHAUER, Querweltein. Das Bild der Evolution als Kollisionsmechanismus des Rechts, in: ZRph $2004,133 \mathrm{ff}$.

103 LUDWIG WITTGENSTEIN, Tractatus logico-philosophicus. Werkausgabe Bd. 1. Frankfurt am Main 1997, Punkte 2.1 bis 3.01

104 JOHANN JACOB BREITINGER, Critische Dichtkunst (1740). Zürich 1966, 334.
} 
tigkeit und Poetik und mit der Zeit fangen Bilder wieder an zu reden und Metaphern zu sehen.

Sind die Bedingungen in der Peripherie juristischer Konflikte dabei anders zu bewerten als im Zentrum? Schon wieder so ein Bild, das von der Architektur des politischen Raums der italienischen Städte lebt und darauf verweist, wie interne und externe Konflikte in den Bedingungen von Differenzierung, Relationierung und Polarisierung zusammenhängen. Dass die Konflikte immer wieder zu einer Herausforderung des decorum führen können, zeigt (ironischerweise?) die Figur des italienischen Professors Giorgio Agamben und seine Schriften. Unabhängig davon, für wie klar oder unklar man die Rechtsphilosophie von Giorgio Agamben hält und für wie tauglich man seine Ausführungen im Diskurs des Rechts erachtet und ob man ihn nur als tropische Figur oder auch seine Texte als Argumente beobachtet: Anselm Haverkamp hat ihm bescheinigt, die "Pole Position" der Philosophie eingenommen zu haben. Im Hinblick auf die Resonanz auf sein Werk erscheint das ganz treffend. ${ }^{105}$ Es gibt Auskunft über die Erhitzung des Bilderstreites im Recht. Auch der Streit um Giorgio Agamben ist der Streit um eine uneigentliche Figur vor dem Hintergrund des eigentlichen Rechts. Mit einer weiteren Ironie ist zu verzeichnen, dass Agamben nicht bloß Jurist ist, sondern in der Öffentlichkeit als Nebenrolle in Pasolinis Verfilmung des Matthäusevangeliums, also in der erhabenen Gattung des Historienbildes, auftauchte. ${ }^{106}$ In seinen Texten wird das decorum des Rechtsstaates wieder explizit, weil auch hier eine erhabene Figur (der homo sacer) auf die Grenzbedingung des Rechts, des Körpers und des Sagbaren bezogen wird. ${ }^{107}$ Die Differenzierung im Medium der Emotion wird synästhetisch wirksam, wenn man sich entscheiden muss, für das Pathos seiner Philosophie empfänglich oder nicht empfänglich zu sein.

Ich sollte vielleicht aufhören, zum Ende zu kommen. Das decorum hat sich oft schon aufgelöst, nie aber sein Ende gefunden, eben weil es Medientechnik ist. Zwischen Recht und dem Rest der Gesellschaft sind immer wieder spontane Restratifikationen, Rehierarchisierungen, funk-

105 Dazu rechtshistorisch RAINER MARIA KIESOW, Ius Sacrum. Giorgio Agamben und das nackte Recht, in: Rechtsgeschichte 1 (2002), 56, und kunsthistorisch MICHAEL F. ZIMMERMANN, Das Bild als Ausnahmezustand. Nancy und Agamben, in: HORST BREDEKAMP / GABRIELE WERNER (Hrsg.), Bildtechniken des Ausnahmezustandes. Münster 2004, 9 und im Übrigen die Übersicht über die Sekundärliteratur zum homo sacer mit über $100 \mathrm{Ti}$ teln auf http://agambeniana.at.infoseek.co.jp/second.html.

106 Agamben spielt dort die Rolle des Phillip, PIER PAOLO PASOLINI, Il Vangelo secondo Matteo (1964).

107 Die Deutung, dass die Figur des homo sacer erhaben und nicht niedrig ist, folgt aus einer schon christlich/spätrömischen Umwertung des Erhabenen durch die Christusfigur, GIORGIO AGAMBEN, Homo Sacer. Die sowveräne Macht und das nackte Leben. Frankfurt am Main 2002. 
tionale Unschärfen und Entdifferenzierungen zu beobachten. In diesen Momenten lässt sich auch beobachten, dass die impliziten Formen des decorum aus den europäischen Gesellschaften nicht verschwunden sind und mit der Vollendung von Zeiträumen auch wieder expliziert werden. Ist es Paranoia, nach weiteren Tradierungen des decorum zu suchen? Dies zu beantworten, zieht noch weiter hinein in die Tiefen des Bilderstreits, weil Paranoia selbst als Polyperspektive eine Technik der Aufmerksamkeit ist. ${ }^{108}$ Jenseits einer funktionalen Ausdifferenzierung des Rechts und jenseits einer medialen Differenzierung von Text und Bild stellt sich die Frage, ob eigentlich über Bilder oder über Recht gestritten wird oder warum im Namen des einen über das andere gestritten wird. Der medientheoretische Verdacht lautet, dass die Erhitzung des Bilderstreites Symptom des Zwanges ist, die Gesellschaft jeweils im Namen des Rechts, im Namen der Kultur oder im Namen der Politik zu identifizieren. ${ }^{109}$ Aber so ist das mit einem Verdacht. Er schafft den paranoiden Blick.

108 „Nur Paranoia fördert Fakten aus der Krypta, dem Unterbau der Archive zu Tage”, WOLFGANG ERNST, Wir vom Archiv, in: Freitag v. 31.08.2001.

${ }^{109}$ Dazu: FABIAN STEINHAUER, Der Rest der Souveränität. Handlungsfähigkeit zwischen Kultur, Recht und Politik, in: BAzON BROCK/GERLINDE KOSCHIK (Mrsg.), Krieg und Kunst. München 2002, 65. 